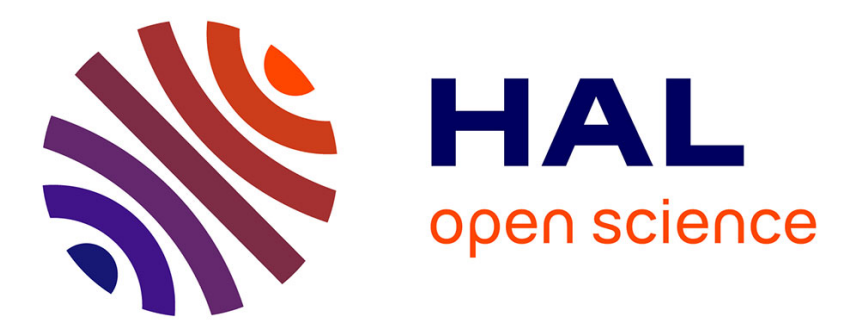

\title{
System dynamic instabilities induced by sliding contact: A numerical analysis with experimental validation
}

\author{
J. Brunetti, F. Massi, Aurélien Saulot, Mathieu Renouf, W d'Ambrogio
}

\section{To cite this version:}

J. Brunetti, F. Massi, Aurélien Saulot, Mathieu Renouf, W d'Ambrogio. System dynamic instabilities induced by sliding contact: A numerical analysis with experimental validation. Mechanical Systems and Signal Processing, 2015, 58-59, pp.70-86. 10.1016/j.ymssp.2015.01.006 . hal-02093229

\section{HAL Id: hal-02093229 \\ https://hal.science/hal-02093229}

Submitted on 8 Apr 2019

HAL is a multi-disciplinary open access archive for the deposit and dissemination of scientific research documents, whether they are published or not. The documents may come from teaching and research institutions in France or abroad, or from public or private research centers.
L'archive ouverte pluridisciplinaire HAL, est destinée au dépôt et à la diffusion de documents scientifiques de niveau recherche, publiés ou non, émanant des établissements d'enseignement et de recherche français ou étrangers, des laboratoires publics ou privés. 


\title{
System dynamic instabilities induced by sliding contact: A numerical analysis with experimental validation
}

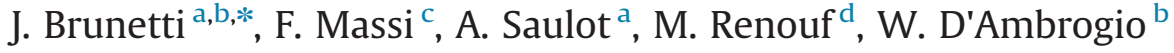 \\ a LaMCoS, Contacts and Structural Mechanics Laboratory, Université de Lyon, CNRS, INSA-Lyon, UMR 5259, 20 rue des Sciences, F-69621, Villeurbanne, France \\ ${ }^{\mathrm{b}}$ DIIIE, Department of Industrial Engineering Information and Economy, Universitá dell'Aquila, Via G. Gronchi 18, I-67100 L'Aquila, Italy ${ }^{\mathrm{C}}$ DIMA, Department \\ of Mechanical and Aerospace Engineering, Universitá di Roma “La Sapienza”, Via Eudossiana, 18, 00184 Roma, Italy ' LMGC, Mechanics and Civil Engineering \\ Laboratory, Université de Montpellier 2, CNRS, UMR 5508, CC048 Place Eugéne Bataillon, \\ F-34096 Montpellier Cedex, France
}

\section{A B S T R A C T}

Mechanical systems present several contact surfaces between deformable bodies. The contact interface can be either static (joints) or in sliding (active interfaces). The sliding interfaces can have several roles and according to their application they can be developed either for maximizing the friction coefficient and the energy dissipation (e.g. brakes) or rather to allow the relative displacement at joints with a maximum efficiency. In both cases the coupling between system and local contact dynamics can bring to system dynamics instabilities (e.g. brake squeal or squeaking of hip prostheses). This results in unstable vibrations of the system, induced by the oscillation of the contact forces.

In the literature, a large number of works deal with such kind of instabilities and are mainly focused on applied problems such as brake squeal noise. This paper shows a more general numerical analysis of a simple system constituted by two bodies in sliding contact: a rigid cylinder rotating inside a deformable one. The parametrical Complex Eigenvalue Analysis and the transient numerical simulations show how the friction forces can give rise to in-plane dynamic instabilities due to the interaction between two system modes, even for such a simple system characterized by one deformable body. Results from transient simulations highlight the key role of realistic values of the material damping to have convergence of the model and, consequently, reliable physical results. To this aim an experimental estimation of the material damping has been carried out. Moreover, the simplicity of the system allows for a deeper analysis of the contact instability and a balance of the energy flux among friction, system vibrations and damping. The numerical results have been validated by comparison with experimental ones, obtained by a specific test bench developed to reproduce and analyze the contact friction instabilities.

Keywords:

Friction-induced vibrations

Mode coupling

Energy balance

Numerical tribology

Damping

\footnotetext{
* Corresponding author at: LaMCoS, Contacts and Structural Mechanics Laboratory, Université de Lyon, CNRS, INSA-Lyon, UMR 5259, 20 rue des Sciences, F-69621, Villeurbanne, France.

E-mail addresses: jacopo.brunetti@insa-lyon.fr (J. Brunetti), francesco.massi@uniroma1.it (F. Massi), aurelien.saulot@insa-lyon.fr (A. Saulot), mathieu.renouf@univ-montp2.fr (M. Renouf), walter.dambrogio@univaq.it (W. D'Ambrogio).
} 


\section{Introduction}

Whenever relative motion between two system components occurs, through a dry contact interface, vibrations are induced by the relative sliding [1]. The local dynamics at the contact (ruptures and wave generation) couples with the system dynamics giving origin to vibrations and regulating the macroscopic frictional behavior of the system [2-4]. The deformations due to the dynamic response of the system affect the local stress distribution; conversely the variation of the local contact stresses and status (sliding, sticking, detachment), the waves generated by the local ruptures, and the local contact characteristics (stiffness, damping, etc.) modify the dynamic response of the system.

Numerical tribology is a powerful tool that developed quickly during the last few decades allowing for a deeper investigation of contact issues [5]. The so-called "friction induced vibrations" [6] can result in either a low amplitude acoustic noise characterized by a large frequency spectrum, which is a function of the surface characteristics (e.g. roughness) and the dynamics of the bodies in contact, or a high amplitude acoustic signal characterized by an harmonic spectrum. The second case is characteristic of dynamic system instabilities excited by the contact forces. Contact dynamic instabilities have been the subject of several analyses focused on specific applications such as joint squeak, squeaking of hip prostheses or brake noises. In particular a large literature can be found on brake NVH (Noise, Vibration and Harshness) issues [7-10] and more specifically on brake squeal [11-20].

A general classification of friction instabilities has been made according to either the frequency of the vibrations or the dynamics of the phenomena [18]. Generally high frequency sound emission is referred as squeal and is characterized by an unstable vibration at an eigenfrequency of the brake system. Nevertheless this kind of harmonic sound emission, due to vibrations excited by sliding contacts, occurs often in commonly used mechanical systems (door hinges, shaft supports, hip endoprosthesis, etc.). Among the mechanisms that are considered responsible for such vibrations, the unstable coupling between two modes of the system is one of the most commonly accepted [21-23,14].

In this work a more general approach is used to highlight this kind of dynamic instability, which is due to the coupling of the tangential and normal deformation at the contact through the friction forces [24,25]. A single deformable solid, a polycarbonate disc, is excited by a rigid disc sliding at its inner radius. In this way any "system" effect, coming from a more complex mechanical system, is avoided and the unstable behavior can be directly related to the dynamics of the single deformable body. Such a simplification allows for a deeper analysis on link between the system dynamic response and the contact behavior during the occurrence of the auto-excited vibrations. First the dynamic instability is reproduced numerically by a linear complex eigenvalues analysis and a nonlinear transient analysis; results are compared to investigate the features of the simulated modal instabilities. The results from the transient simulations highlight the need of realistic material damping coefficients retrieved by experimental modal tests to obtain reliable results. Once the material damping is measured, the energy balance of the system during the unstable vibrations is presented and the relative role on the braking energy dissipation due to either friction and material damping is discussed. A parametrical analysis is also developed to investigate the role of the friction coefficient and the sliding velocity. Finally experimental results are presented to validate the numerical ones. Complementary analyses are focused on experimental observations $[26,27]$ and numerical simulations of third body flows [28-30] at the contact and its effect on the onset of dynamic instabilities [31].

\section{The model}

The system considered for this work is composed of a deformable disc made of polycarbonate, which is constrained at its external circumference. A coaxial cylinder made of steel is put in rotation inside the polycarbonate one; a static radial expansion of the steel cylinder ensures the contact with the inner circumference of the polycarbonate disc, imposing the wished contact pressure. Radial forces (normal to the contact surface) and friction forces (along the tangential direction) at the contact interface between the two discs induce vibrations on the system.

\subsection{Experimental setup}

The polycarbonate disc (inner radius $20.5 \mathrm{~mm}$, external radius $90.5 \mathrm{~mm}$, thickness $9 \mathrm{~mm}$ ) is clamped on a rigid frame, and a steel cylinder is linked by a transmission belt to the driving motor (cf. Fig. 1(a)). Inside the steel cylinder a piston produces the axial compression of an elastic component (rubber cylinder placed inside the steel one) that tries to expand radially and generates the radial expansion of the outer steel cylinder; the radial expansion is measured by an inductive proximity sensor and stopped when the wished contact pressure between the two bodies is reached. A full description of the expanding chuck and of the precautions adopted to minimize the axial asymmetry and conicity error is reported in $[26,27]$. The steel cylinder is maintained at constant rotational speed by a controller and the engine torque is measured during tests.

Experimental measurements on quantities inside of the contact are quite difficult: significant errors can arise due to the introduction of sensors and consequent modification of the contact pair. This is why the numerical simulation is preferred to investigate the issue. In this work the experimental set-up showed in Fig. 1(a) is used to validate the numerical analysis results by comparison of the macroscopic dynamic behavior of the system. To this aim, accelerometers have been placed on the free surface of the polycarbonate disc to measure the system vibration during sliding and compare the acceleration 
a

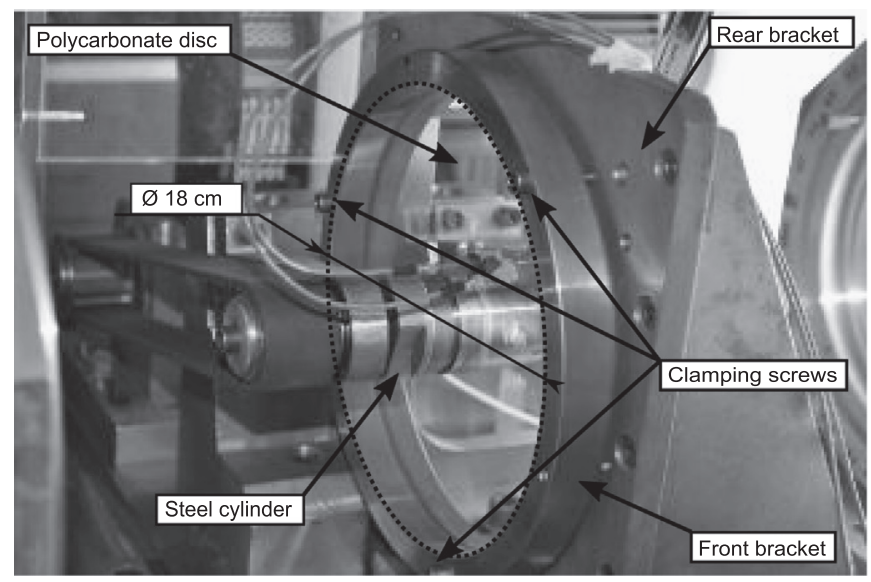

b

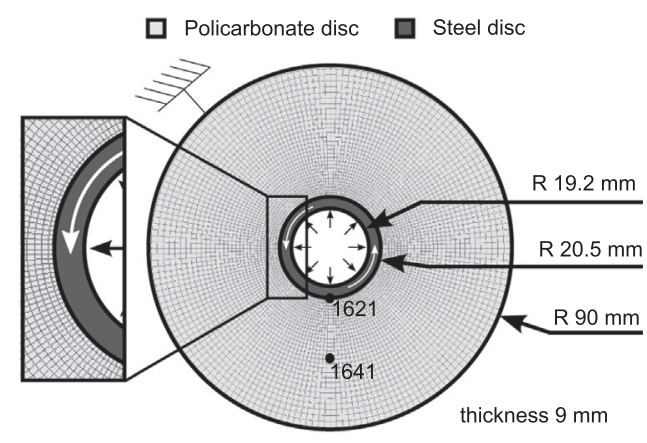

Fig. 1. Experimental and numerical model. (a) The experimental setup. PhotoTrib. (b) 2D geometrical and finite element model for numerical simulation.

signals with the numerical ones. The polycarbonate material has been chosen for its photoelastic properties and transparency for the observation of shear stress distribution and third body analysis, reported in [26,32].

\subsection{Numerical model}

To analyze and reproduce numerically the vibrational behavior of the polycarbonate disc, two different approaches are used. A linear finite element model is used with the commercial code ANSYS to perform a parametrical prestressed Complex Eigenvalue Analysis (CEA) of the system. A non-linear finite element model is used with the code PLAST2 [24,33,34] to perform a transient analysis in explicit dynamic formulation with the aim to reproduce numerically the friction induced vibrations observed experimentally and predicted by the CEA.

In the presented analyses a simplified 2D finite element model with a plane strain formulation is considered. Nevertheless a prior analysis on a full 3D model, including the frame of the mechanical system, has been developed and has highlighted that the dynamic instabilities of interest are characterized by in-plane vibrations; thus they can be observed as well by the 2D model with a substantial reduction of the computational time. A comparison between the in-plane mode frequencies calculated by the full 3D model and the simplified 2D model showed that frequencies of the simplified 2D model are slightly higher than the frequencies of the full 3D model with a percentage error less than $7.5 \%$ in the frequency range of interest. This difference in frequency does not affect the modal shape that is very similar between the two modes. Furthermore, the difference in frequency can be related to mainly three reasons: (i) the introduction of a fixed boundary on the external edge of the 2D model, instead of the external frame, which increases the stiffness of the system; (ii) the mass of the external frame is not taken into account in the 2D model that results to be less massive than the 3D model and the real system; (iii) the plane strain formulation introduces an out of plane constraint that increases the global stiffness of the system.

Moreover, a further difference with the experiments is due to the connection between the polycarbonate disc and the frame: while the connection is bounded in the 3D numerical model, three screws and a steel annular frame are used to clamp the disc in the experiments. The rigidity of the experimental connection is lower explaining the lower mode frequencies. The simplified boundary conditions and the 2D model produce an acceptable overestimation of the in plane frequencies; these considerations have to be accounted for when comparing the experimental and numerical results (cf. Section 6).

The difference in stiffness between the materials of the two cylinders in contact (the polycarbonate Young's modulus is about $2.5 \mathrm{GPa}$ while that of steel is about $210 \mathrm{GPa}$ ) allows for considering only one deformable body (the polycarbonate disc) in contact with a rigid one (the steel cylinder).

The geometry of the 2D simplified model used for numerical simulations is reported in Fig. 1(b). The external circumference of polycarbonate disc is constrained to the fixed frame and the inner cylinder is in rotation with respect to the external one. To obtain the desired radial contact pressure the inner cylinder is expanded as in the case of the experimental test. The radial expansion applied numerically is $25 \mu \mathrm{m}$.

Convergence analyses have been performed to find an optimal mesh size both for the linear model in view of the complex modal analysis and for the non-linear model to perform the transient simulations. The finite element model adopted for modal analysis is composed of 2038 elements with a size of the contact elements of $0.859 \mathrm{~mm}$. The model adopted for nonlinear transient analysis is composed of 6300 elements with a size of the contact elements of $0.715 \mathrm{~mm}$ (cf. Fig. 1(b)). Labeled dots indicate the position of the contact node 1621 and of the internal node 1641 considered in the transient simulations (Section 5). 


\section{Material damping}

System motion can be expressed as linear combination of eigenmodes of the system. If there is material damping in the system each eigenmode is characterized by a modal damping, generally positive (corresponding to a negative real part of the eigenvalue), that brings to a progressive decrease of the mode vibration.

In the mathematical model of mechanical systems without contact, stiffness and mass matrix are generally symmetric. In this case it is possible to diagonalize the system:

$$
[M]\{\ddot{x}\}+[C]\{\dot{x}\}+[K]\{x\}=\{0\},
$$

considering a proportional damping formulation:

$$
[C]=\alpha_{R}[M]+\beta_{R}[K] .
$$

Damping factor can be expressed as a function of the angular frequency $\omega$ :

$$
\zeta=\frac{1}{2}\left(\frac{\alpha_{R}}{\omega}+\beta_{R} \cdot \omega\right) .
$$

The Rayleigh coefficients $\alpha_{R}$ and $\beta_{R}$ affect the proportional damping respectively at the low frequencies and at the high frequencies. The system eigenvalues represented in the complex plane lie on a parabolic curve on the negative real part halfplane:

$$
\lambda=-\zeta \omega \pm i \omega \sqrt{1-\zeta^{2}}
$$

and

$$
\operatorname{Re}(\lambda)=-\frac{\alpha_{R}}{2}+\frac{2 \beta_{R} \omega^{2} \cdot \operatorname{Im}(\lambda)^{2}}{\alpha_{R}^{2}-2\left(2+\alpha_{R} \beta_{R}\right) \omega^{2}+\beta_{R}^{2} \omega^{4}}
$$

A convergence analysis over space and time discretization has been performed and highlighted the main role played by the damping coefficients to obtain the convergence of results in transient numerical analysis.

Shear stress fields reported in Fig. 2 show that completely different transient behaviors can be obtained for different values of the material damping coefficients.

For low values of Rayleigh coefficients (cf. Fig. 2(a)), the system presents an unstable vibrational behavior, but no convergence in solution can be reached increasing the number of elements or reducing the time step for the calculation of transient solution. In this case the response of the system is not harmonic and the in plane vibration results to be different from any the modal shape found in the CEA.

On the contrary, for high values of the damping coefficients $\alpha_{R}$ and $\beta_{R}$ no convergence problems arise, but the system is always stable (cf. Fig. 2(c)). In this case, the system reaches a static equilibrium position with all the nodes over the contact interface in a uniform sliding status.

When introducing realistic values of material damping coefficients (cf. Fig. 2(b)), calculated experimentally, the system vibrates at a frequency that is close to one of the unstable modes predicted by the CEA and the velocity field results to be similar to the modal shape of the unstable mode. The same unstable mode is retrieved experimentally (cf. Fig. 15 and Section 6).

Focusing on the energy flows through the deformable body in the case of too low damping values, the energy introduced into the system by frictional forces cannot be dissipated correctly by the internal non-conservative damping forces, so that the internal energy increases; the waves generated at the contact are continuously reflected at the boundaries (cf. Fig. 2(a)). This nonequilibrium can be considered at the origin of the non-convergence of the solution. Increasing the damping coefficient values, the energy equilibrium can be reached and, for very high values of the damping coefficients, the system becomes artificially stable because the energy is rapidly dissipated by the damping forces.
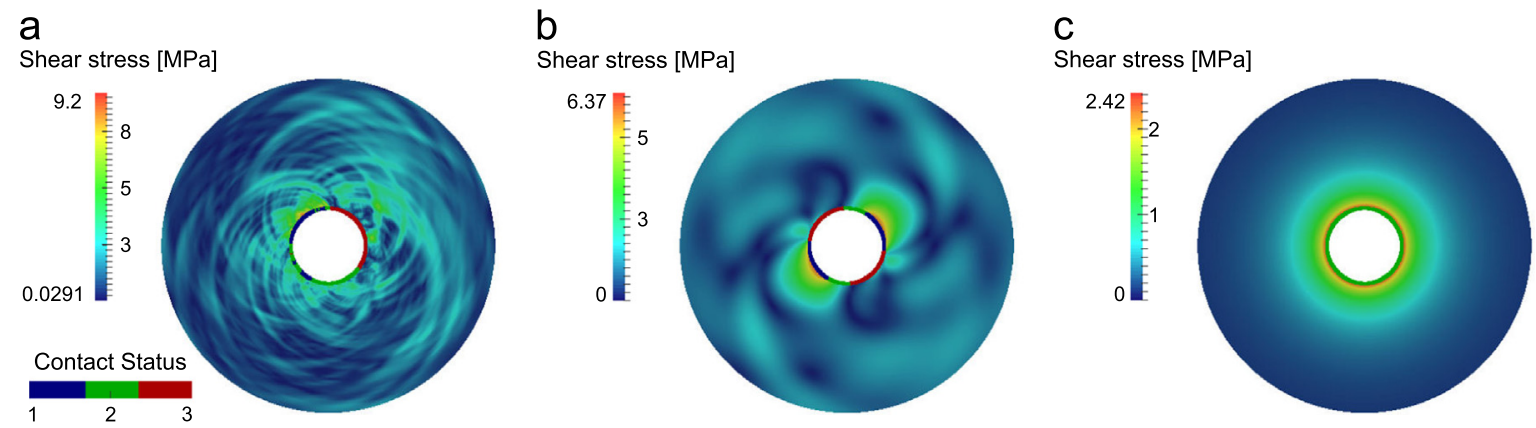

Fig. 2. Different behavior (shear stress (MPa)) of the system depending on the material damping coefficients. (a) $\alpha_{R}=101 / \mathrm{s} ; \beta_{R}=1 E-9 \mathrm{~s}$. (b) $\alpha_{R}=401 / \mathrm{s}$; $\beta_{R}=4.5 E-7 \mathrm{~s}$. (c) $\alpha_{R}=1001 / \mathrm{s} ; \beta_{R}=1.2 E-6 \mathrm{~s}$. (For interpretation of the references to color in this figure caption, the reader is referred to the web version of this paper.) 
a
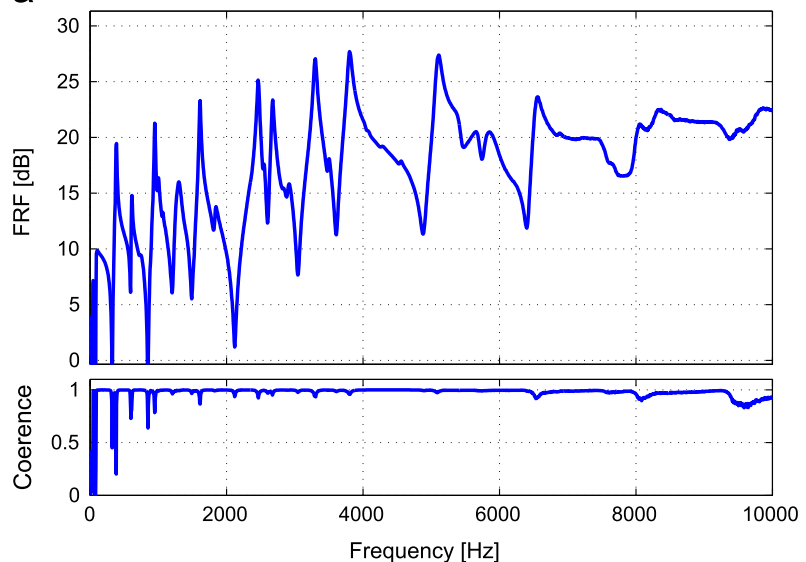

b

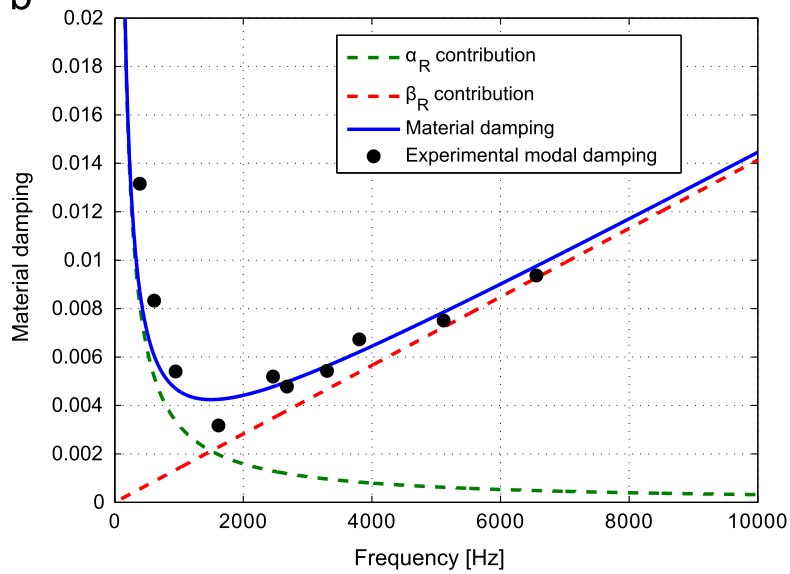

Fig. 3. Experimental Rayleigh coefficient identification. (a) Experimental FRF, and (b) modal damping and damping coefficient identification. (For interpretation of the references to color in this figure caption, the reader is referred to the web version of this paper.)

This means that the material damping introduced into the system drastically affects the numerical solution that can be non-convergent or give unreliable results. Because of its key role, the material damping of the polycarbonate disc has been estimated experimentally. An experimental modal analysis has been performed on the disc to estimate the modal damping factors by the half power method.

Fig. 3(a) presents the Frequency Response Function (FRF) obtained by exciting the disc with an instrumented hammer in free-free conditions and measuring the response with an accelerometer. The half power method has been used to calculate the modal damping factors for each mode in the frequency range 0-10 $000 \mathrm{~Hz}$ (black marks in Fig. 3(b)). By comparison between the experimental modal damping factors and the damping frequency behavior obtained with the proportional damping matrix (blue continuous line), the values of the Rayleigh coefficients are calculated. Fig. 3(b) shows the contributions of the $\alpha_{R}$ Rayleigh coefficient (green dashed line) and the $\beta_{R}$ coefficient (red dashed straight line) to the numerical material damping.

The Rayleigh coefficients obtained are $\alpha_{R}=401 / \mathrm{s}$ and $\beta_{R}=4.5 E-7 \mathrm{~s}$. As expected, with these values the convergence of the model is obtained and an unstable modal vibration of the system, corresponding to the experiments (see Section 6 ), is simulated by the numerical transient analysis.

\section{Instability analysis and modal coupling}

One of the most accredited mechanisms at the origin of system instabilities due to frictional contact is the modal coupling or mode lock-in [1,23,35]. Different numerical and experimental works [14,15,11], developed on complex systems with two or more deformable bodies in contact, highlighted that the unstable behavior arises when the frictional forces cause the coalescence between pairs of modes of the different system components.

By the Complex Eigenvalue Analysis (CEA) it is possible to find the complex eigenvalues and eigenvectors of the system. A pre-stress quasi-static analysis is performed to apply the contact forces in sliding condition: first an expansion of the inner cylinder of $25 \mu \mathrm{m}$ is applied to ensure the contact pressure; then the inner disc is put in rotation to bring the whole contact surface in sliding. Frictional forces acting at the contact interface are linearized for the Complex Eigenvalue Analysis by introducing normal and tangential stiffness at the contact elements that results as asymmetries on the stiffness matrix. Afterwards, a complex modal analysis is performed on the model, which is linearized around the equilibrium position in sliding condition.

Proportional damping has been considered in this calculation and the experimental values for Rayleigh coefficients (cf. Fig. 3(b)) have been introduced. The resulting complex eigenvalues give information on the frequency of the modes of vibration and on the modal damping (see Eq. (4)). Eigenvalues with positive real part (negative modal damping) correspond to unstable modes of the mechanical system. In fact expressing the system motion as linear combination of all the system modes, there are positive exponential terms that turn the system away from the equilibrium position generating an unstable vibration at the frequency of the mode with positive real part.

Increasing the frictional forces (i.e. increasing the friction coefficient) affects the system by modifying the real parts of the coalescing modes which depart from the original one due to the material damping. One of the two coalescing eigenvalues moves toward the positive half space of the real part. When it becomes positive it causes the instability of the system.

In a mechanical system, each mode of the coalescing pair involves the deformation of the different components of the system i.e. the deformation of both the bodies in contact should be accounted for. In this work the system is composed of only one deformable body (polycarbonate disc) in frictional contact with a rigid surface. Also in this case the friction forces introduce an asymmetry in the stiffness matrix and couple the tangential and normal deformation of the modes at the 
contact surface allowing for some mode to become unstable. All the eigenmodes of the systems belong in this case to the external polycarbonate disc. Thus the unstable coupling occurs between modes of vibration of the same component.

\subsection{Complex Eigenvalue Analysis (CEA)}

Results reported here are referred to a friction coefficient at the contact $\mu=0.3$. Table 1 reports the eigenvalues of the polycarbonate disc resulting from the prestressed CEA.

Fig. 4 shows the first modes resulting from the CEA. Modal shapes are represented in color scale, red zones correspond to the zones of polycarbonate disc with highest displacement with respect to the equilibrium position. Modes $1,8,19$ and 24 are pure radial or pure torsional modes. Conversely due to the symmetry of the system all the others are coupled and have two by two the same frequency. In the absence of friction the double modes would be identical (same frequency and same modal damping), on the contrary the asymmetry introduced by the frictional interaction causes the two modes to be slightly different in terms of vibration frequency and significantly different in terms of modal damping.

The unstable modes are 3, 5, 7 and 26 at respectively $7.66 \mathrm{kHz}, 9.90 \mathrm{kHz}, 10.53 \mathrm{kHz}$ and $17.19 \mathrm{kHz}$. The deformed shape of the unstable modes shows a large deformation at the contact area (see red and yellow zones on modes 2-3, 4-5 and 6-7 in Fig. 4), where the contact forces allow for the coupling. Coupled modes that are not unstable are characterized by an irrelevant deformation at the contact (see modes 9-18 in Fig. 4).

Table 1

Complex eigenvalues for $\mu=0.3$, and with the damping coefficients identified experimentally. Gray highlight: unstable eigenvalues; Bold: uncoupled eigenvalues.

\begin{tabular}{lccr}
\hline Mode & Compl. Eigenvalue $\mathbf{( H z )}$ & Mode & Compl. Eigenvalue (Hz) \\
\hline $\mathbf{1}$ & $-\mathbf{5 1 . 7} \pm \mathbf{5 8 5 7 i}$ & 15 & $-318.8 \pm 14673 \mathrm{i}$ \\
2 & $-207.1 \pm 7655 \mathrm{i}$ & 16 & $-296.7 \pm 14674 \mathrm{i}$ \\
$\mathbf{3}$ & $34.9 \pm 7660 \mathrm{i}$ & 17 & $-366.7 \pm 14777 \mathrm{i}$ \\
4 & $-438.6 \pm 9884 \mathrm{i}$ & 18 & $-257.5 \pm 14781 \mathrm{i}$ \\
5 & $155.7 \pm 9901 \mathrm{i}$ & $\mathbf{1 9}$ & $-\mathbf{3 1 4 . 3} \pm \mathbf{1 4 8 3 2 i}$ \\
6 & $-556.3 \pm 10502 \mathrm{i}$ & 20 & $-726.9 \pm 16345 \mathrm{i}$ \\
$\mathbf{8}$ & $237.8 \pm 10525 \mathrm{i}$ & 21 & $-36.4 \pm 16377 \mathrm{i}$ \\
9 & $-\mathbf{1 7 6 . 7} \pm \mathbf{1 1 0 7 7 i}$ & 22 & $-383 \pm 16433 \mathrm{i}$ \\
10 & $-330.9 \pm 11258 \mathrm{i}$ & 23 & $-\mathbf{3 9 4 . 9} \pm \mathbf{1 6 6 4 1 i}$ \\
11 & $-34.1 \pm 11268 \mathrm{i}$ & $\mathbf{2 4}$ & $-842.6 \pm 17145 \mathrm{i}$ \\
12 & $-281.8 \pm 11770 \mathrm{i}$ & 25 & $3.1 \pm 17186 \mathrm{i}$ \\
13 & $-116.5 \pm 11775 \mathrm{i}$ & 26 & $-500.1 \pm 17444 \mathrm{i}$ \\
14 & $-285.7 \pm 12915 \mathrm{i}$ & 27 & $-367.5 \pm 17451 \mathrm{i}$ \\
\hline
\end{tabular}
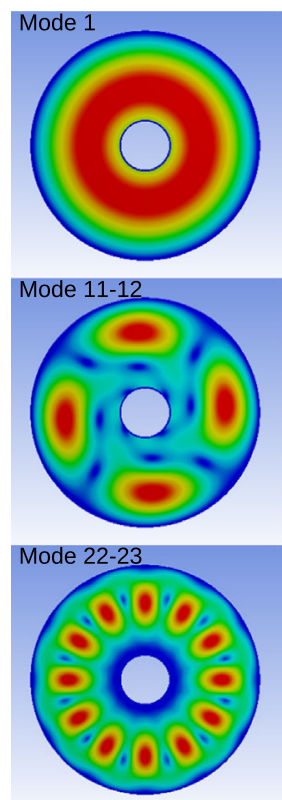
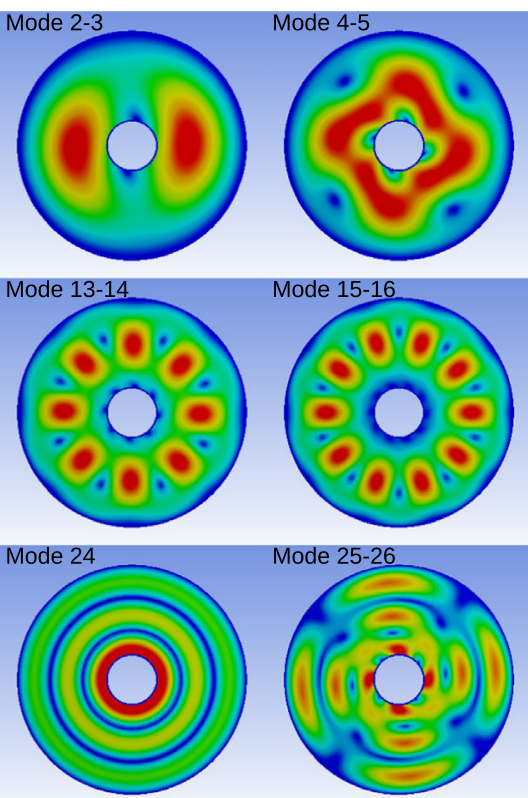
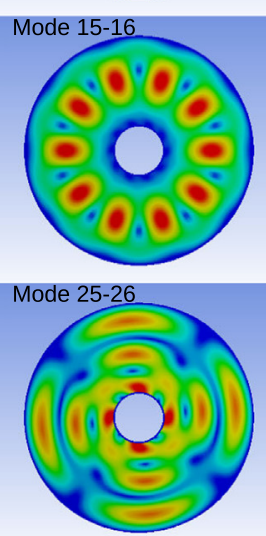
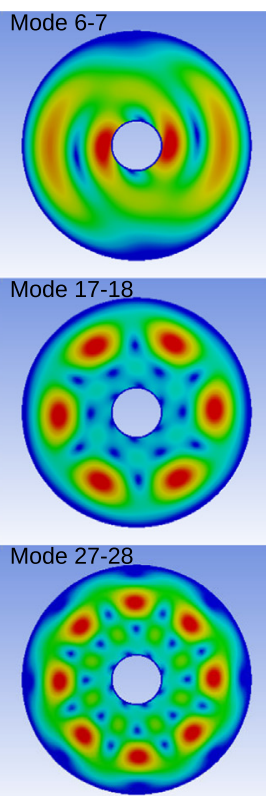
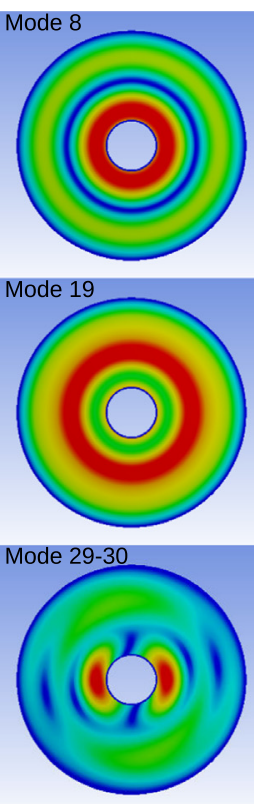
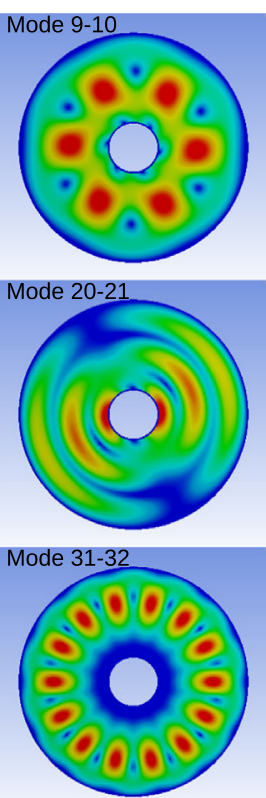

Fig. 4. First modal shapes resulting from the CEA with a friction coefficient $\mu=0.3$. (For interpretation of the references to color in this figure caption, the reader is referred to the web version of this paper.) 


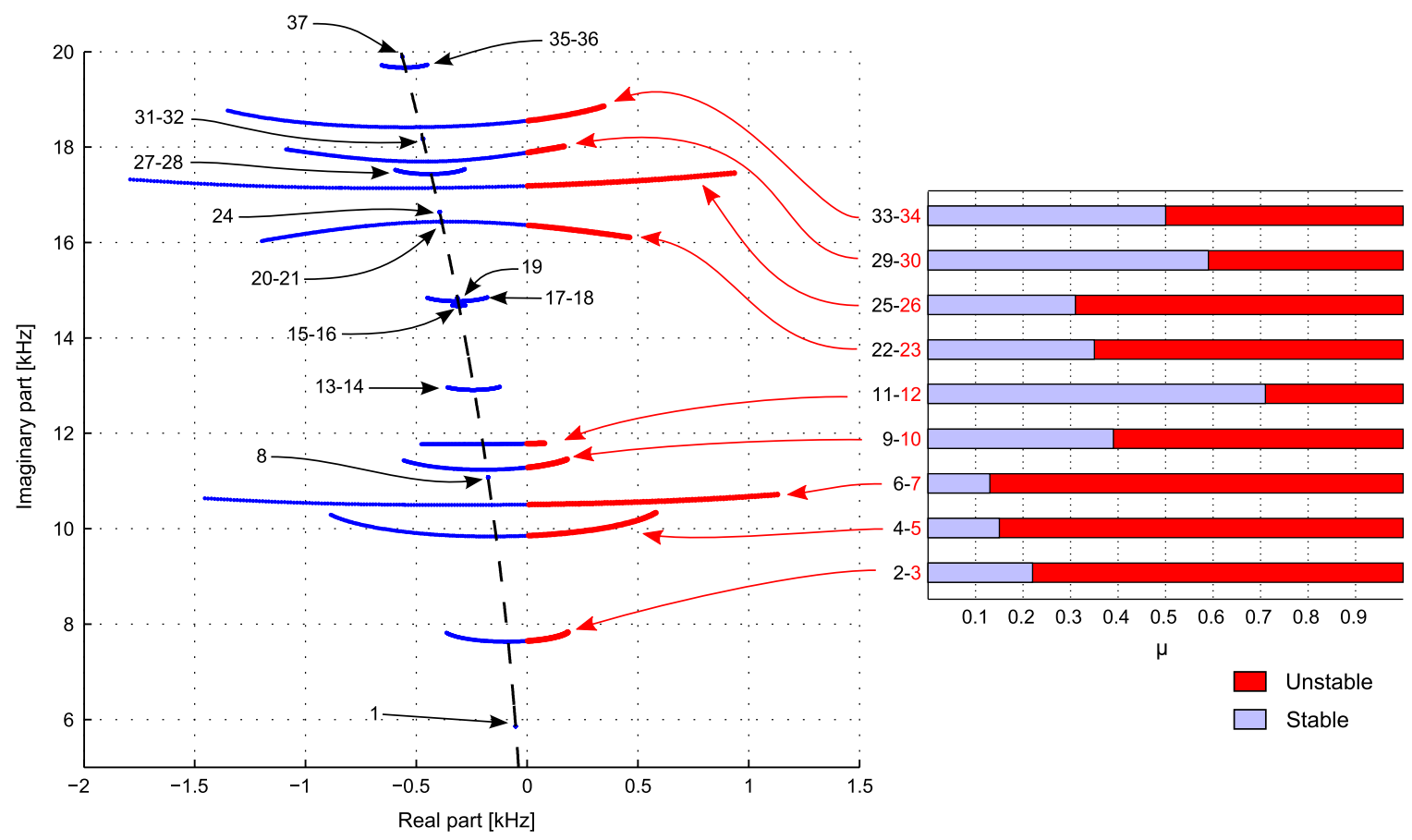

Fig. 5. Locus plot of the system eigenvalues. For each eigenvalue that becomes unstable the critical value of the friction coefficient is reported in the stability plot. (For interpretation of the references to color in this figure caption, the reader is referred to the web version of this paper.)

\subsection{Effect of local friction on the system dynamics}

The stability of a linear system can be evaluated basically by the observation of the real part of the eigenvalues of the system resulting from the complex modal analysis. In stability verification the friction coefficient largely affects the results because it modifies the asymmetry introduced into the stiffness matrix. In Fig. 5 are reported the system eigenvalues on the complex plane obtained when changing the friction coefficient $\mu$ in the range from 0 to 1 .

The dashed curve in Fig. 5 represents the theoretical locus of the eigenvalues of the damped system. The two coalescing eigenvalues lie on this curve and are coincident for a nil friction coefficient. Increasing the friction coefficient they are split in opposite directions and the real part of one mode of the pair can become positive i.e. unstable (red points on the locus plot in Fig. 5). This phenomenon, known in brake squeal literature as lock-in or modal coupling, is here obtained for pairs of modes belonging to the same deformable body. The bar diagram on the right of Fig. 5 shows the instability intervals (red bars) of friction coefficient for coupled modes that become unstable.

Comparison between the modal shapes in Fig. 4 and the result of parametric stability analysis in Fig. 5 highlights that modes having large deformation at the contact zone (modes 3, 5 and 7) become unstable; modes with small deformation at the contact zone (modes 14,16 and 18) are slightly affected by increasing the friction coefficient and remain stable. Mode 7 is the first mode that reaches the instability by increasing the friction coefficient and it is characterized by the highest displacement over the contact interface (length of the red zones over the contact interface in Fig. 5).

Fig. 6 shows the behavior of the mode pairs $4-5$ and 6-7, at about $9.85 \mathrm{kHz}$ and $10.5 \mathrm{kHz}$, in terms of frequency $f$ and modal damping factor $\zeta$ (see Eq. (4)) with respect to the friction coefficient. Red points correspond to unstable mode (negative values of the modal damping). For the 7th mode the critical value of the friction coefficient is $\mu_{7 c}=0.13$, while for the 5th mode the critical value is $\mu_{5 c}=0.15$. In brake squeal literature the coupling occurs between eigenfrequencies that coalesce with the increase of the friction coefficient, even if they have different frequencies for a nil friction coefficient [23]. The system investigated here is completely axial-symmetric and the two coalescing modes have the same frequency when friction coefficient is nil. For this reason the increase of friction coefficient causes a small shift between the mode frequencies (left plot in Fig. 6).

The real parts (cf. Fig. 5) and the modal dampings (right plot in Fig. 6) of the eigenvalue pair split toward the two opposite half spaces; the behavior, increasing the friction coefficient, is symmetric with respect to the starting value because the two modes have equal modal damping (they belong to the same body with the same material damping) and same deformed shape. It is worth to notice that, between the two modes of the coalescing pair, the mode that becomes unstable is the higher frequency one and this result is here obtained for all the coalescing pairs.

Fig. 7 shows the mode 7 for different values of the friction coefficient $\mu$. Increasing the friction forces the asymmetry on the system matrices modifies the modal shape with respect to the mode without friction. This effect results to be as much relevant as the distribution of the deformation at the contact is relevant. 
a

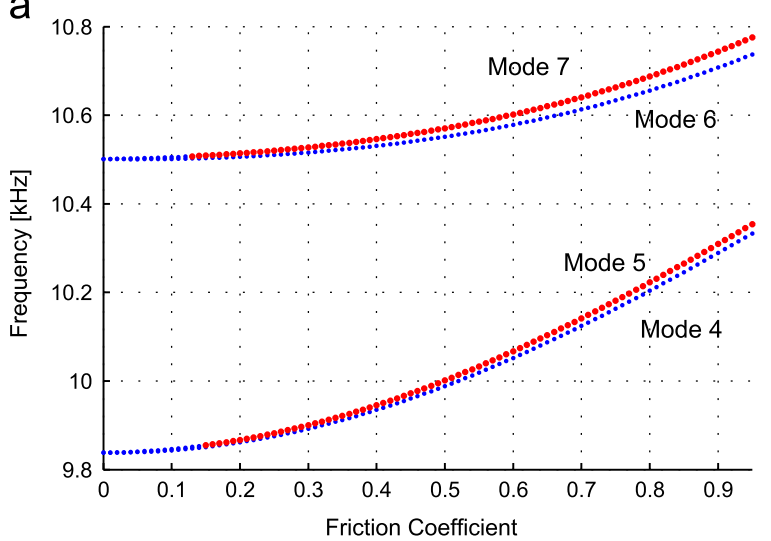

b

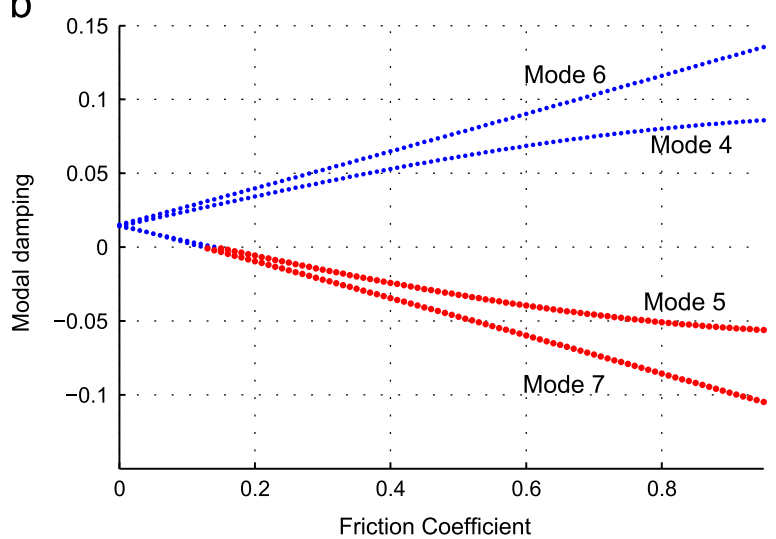

Fig. 6. Frequency and modal damping of the first unstable modes for different friction coefficients. (For interpretation of the references to color in this figure caption, the reader is referred to the web version of this paper.)

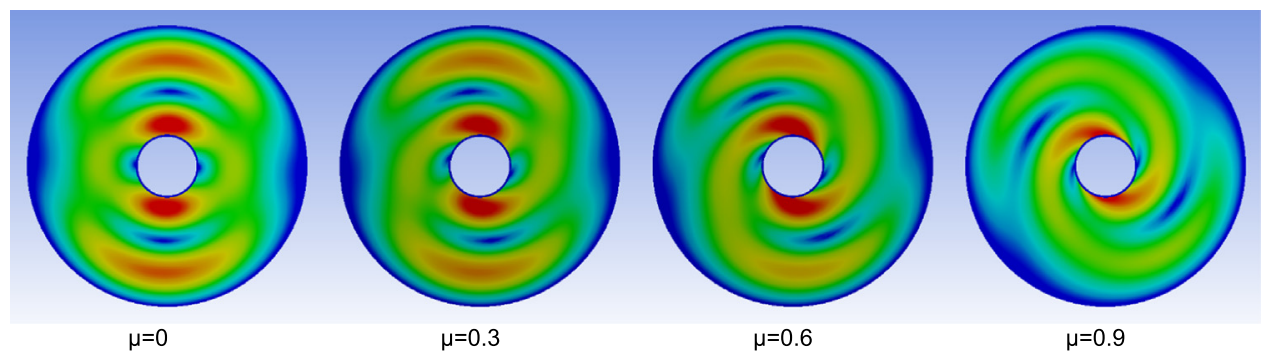

Fig. 7. Effects of the friction coefficient on the 7th mode of the polycarbonate disc. (For interpretation of the references to color in this figure caption, the reader is referred to the web version of this paper.)

\section{Transient analysis}

To reproduce numerically the dynamic response of the polycarbonate disc during sliding a nonlinear model has been developed. To simulate the nonlinear frictional contact the dynamic finite element code PLASTD is used.

This code uses a forward incremental Lagrange multiplier method $[36,24]$ to evaluate the normal and tangential contact stresses as well as the contact status (stick, slip or detachment). The code uses an explicit formulation to solve the transient problem. The forward incremental Lagrange multiplier method equations set is constructed using the equations of motion developed via the principle of virtual work at time step $t_{n}$ augmented by displacement constraints acting on contacting surfaces at time $t_{n+1}$ :

$$
\left\{\begin{array}{l}
{[M]\{\ddot{u}\}_{n}+[C]\{\dot{u}\}_{n}+[K]\{u\}_{n}+[G]_{n+1}^{T}\{\lambda\}_{n}=\{F\}_{n}^{e x t}} \\
{[G]_{n+1}^{T}\left[\{X\}_{n}+\{u\}_{n+1}-\{u\}_{n}\right] \leq 0}
\end{array}\right.
$$

where $\{\lambda\}_{n}$ are the contact forces acting on the contact nodes introduced as Lagrange multipliers. $[G]_{n+1}$ is a collocation matrix of the contact forces over the degrees of freedom of the whole mechanical system. $\{X\}_{n+1}=\{X\}_{n}+\{u\}_{n+1}-\{u\}_{n}$ is the coordinate vector at time $n+1 .\{u\}_{n},\{\dot{u}\}_{n}$ and $\{\ddot{u}\}_{n}$ are respectively the displacement, the velocity and the acceleration of the degrees of freedom of the system. $[M],[C]$ and $[K]$ are the mass, damping and stiffness matrices of the system respectively.

This equation is then discretized over time for the transient simulation. The explicit integration scheme is the Newmark scheme:

$$
\left\{\begin{array}{l}
\{\ddot{u}\}_{n}=\frac{2}{\Delta t^{2}}\left(\{u\}_{n+1}-\{u\}_{n}-\Delta t \cdot\{\dot{u}\}_{n}\right) \\
\{\dot{u}\}_{n}=\frac{1}{1+2 \beta_{2}}\left[\{\dot{u}\}_{n-1}+\Delta t\left(1-\beta_{2}\right)\{\ddot{u}\}_{n-1}+\frac{2 \beta_{2}}{\Delta t}\left(\{u\}_{n+1}-\{u\}_{n}\right)\right]
\end{array}\right.
$$

In this formulation the coefficient $\beta_{2}$ is related to the numerical damping and when it is equal to 0.5 this method corresponds to the central differences method and does not introduce any numerical damping. For our analysis this value will be adopted with the aim of not introducing non-physical damping into the system. The energy balance during the 

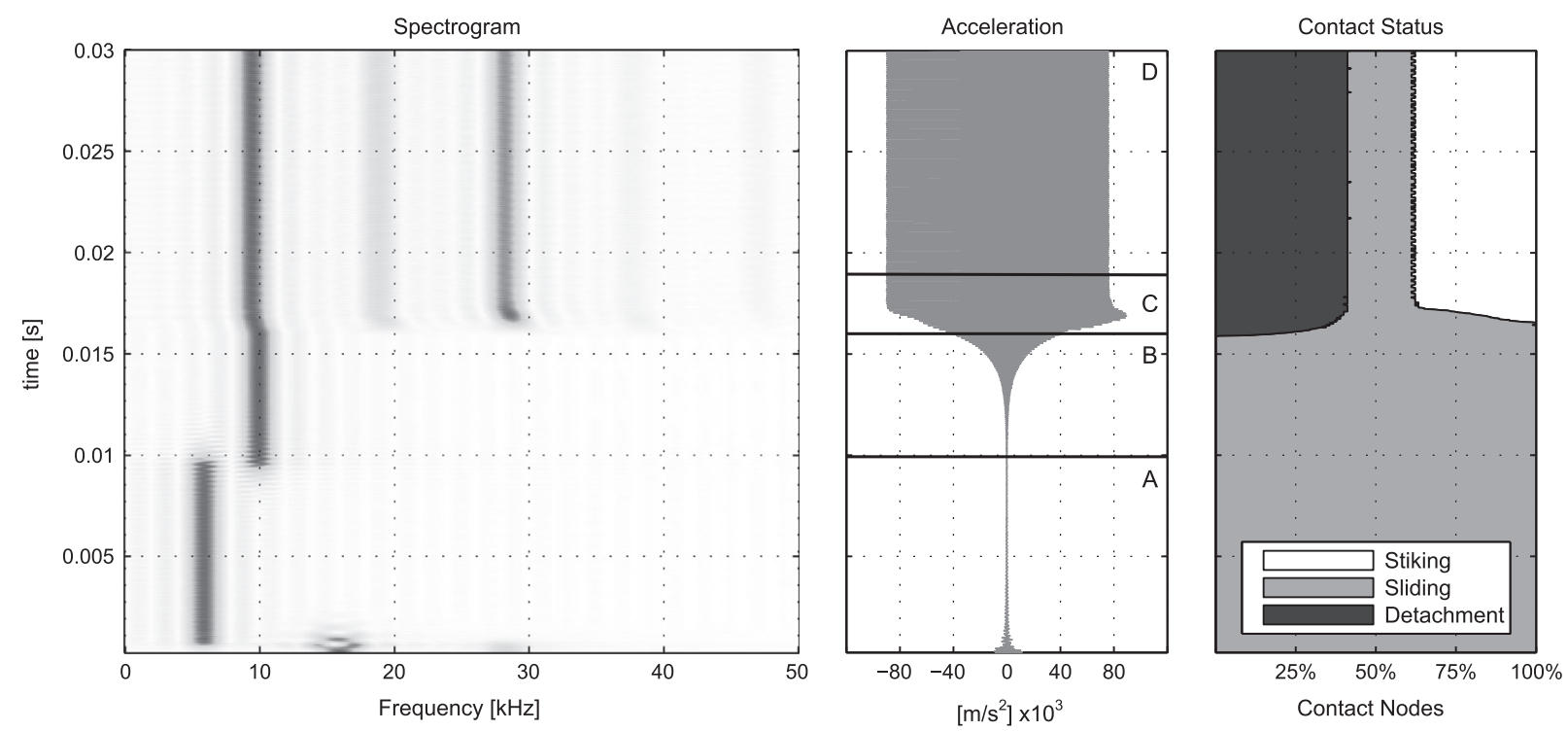

Fig. 8. Acceleration of an internal node with its spectrogram and status of the contact nodes over the time. (For interpretation of the references to color in this figure caption, the reader is referred to the web version of this paper.)

a

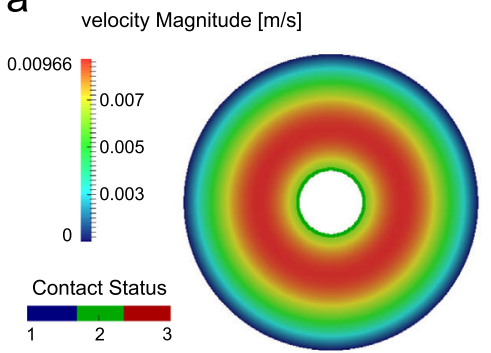

b

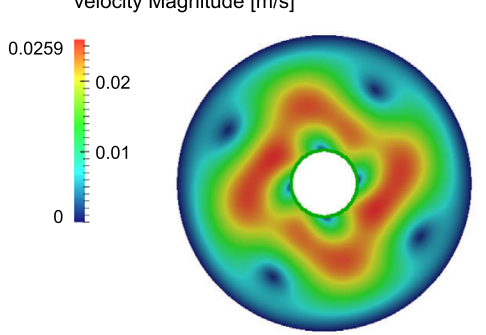

C velocity Magnitude $[\mathrm{m} / \mathrm{s}]$

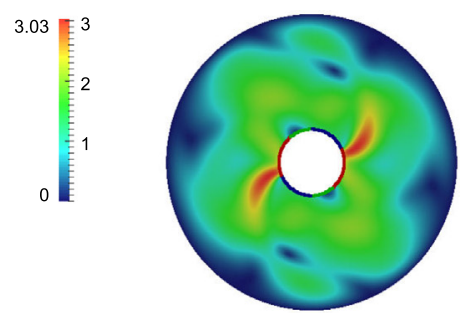

Fig. 9. Velocity field (m/s) and contact status at different times. (a) $t=0.005 \mathrm{~s}$, (b) $t=0.012 \mathrm{~s}$, and (c) $t=0.030 \mathrm{~s}$.

transient simulation will be guaranteed by the material damping of the polycarbonate expressed as proportional Rayleigh damping.

\subsection{Transient analysis results for $\mu=0.3$ and $\omega=600 \mathrm{rpm}$}

Hereafter, results from a transient analysis performed with the same boundary conditions imposed for the CEA are reported.

The inner disc has an imposed rotational speed $\omega=610 \mathrm{rpm}$; the inner disc is expanded of $25 \mu \mathrm{m}$ to engage the contact with polycarbonate disc. The friction coefficient is set to 0.3 and the Rayleigh coefficients are equal to the experimental values $\left(\alpha_{R}=401 / \mathrm{s}\right.$ and $\left.\beta_{R}=4.5 E-7 \mathrm{~s}\right)$.

Observing the acceleration response of a node of the disc (cf. Fig. 8), four different stages can be highlighted and identified by the letters from A to D. In the leftmost plot in Fig. 8 the spectrogram of the acceleration response highlights the different harmonic contents of each stage of simulation. The reported spectrogram is calculated with a window length of $0.5 \mathrm{~ms}$. The amplitude of each FFT calculated for building the spectrogram is normalized, so that the maximum values of each FFT are the same (although the vibration amplitude changes largely) and they can be plotted on the same diagram giving information about the evolution of the main harmonic content for the whole simulation. Furthermore the status of contact nodes over the time (cf. rightmost plot in Fig. 8) shows the effect of contact status variation on the transient behavior of the system.

The first stage of the transient response (stage A in Fig. 8) is characterized by a small amplitude of vibration excited by the rapid expansion of the rigid surface, which gives an impulsive radial and rotational excitation to the disc. The disc response at a frequency of $5.8 \mathrm{kHz}$ corresponds to the first (radial) vibration mode of the system (see Table 1). All the contact nodes are in sliding status and the vibration amplitude decreases according to the sign of the real part of the first eigenvalue. Fig. 9(a) shows the scalar field of in plane velocity modulus for $t=0.005 \mathrm{~s}$, which correspond to a radial mode of vibration of the disc. This velocity distribution results to be similar to the mode 1 in Fig. 4. 


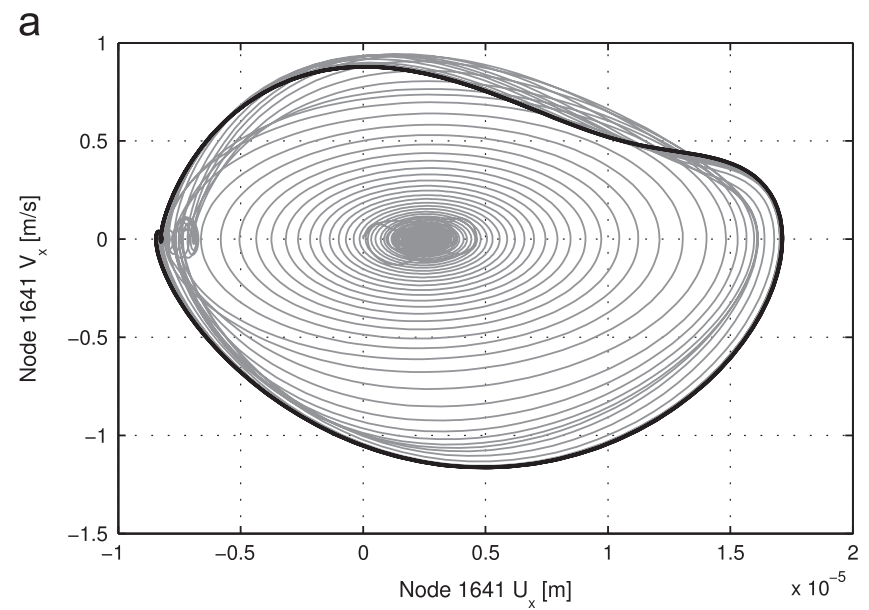

b

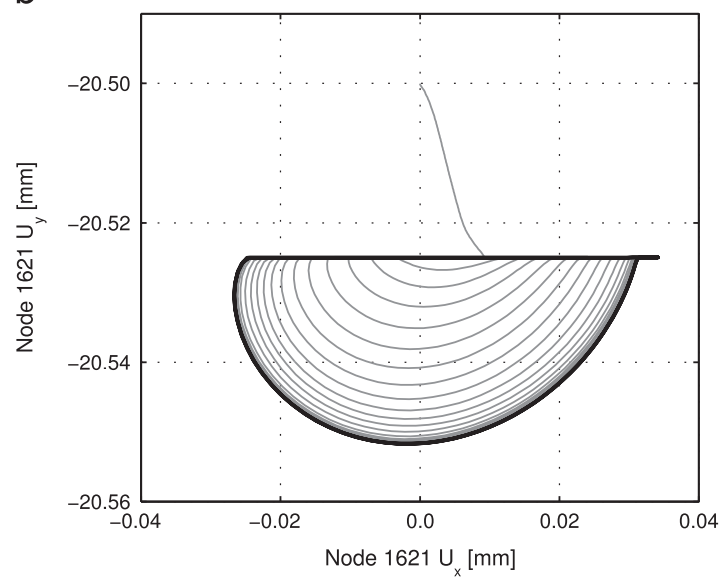

Fig. 10. Limit cycles. (a) Displacement vs. speed on the 1641 node in the $x$ direction. (b) Trace of the contact node 1621 .

At time $t \simeq 0.01 \mathrm{~s}$ the spectrogram in Fig. 8 presents a switch in the main frequency of vibration. From this point forward (stage B) the main harmonic content is at a higher frequency of about $9.9 \mathrm{kHz}$ that corresponds to the mode 5, which is predicted to be unstable by the CEA. The vibration amplitude grows exponentially, which is typical of unstable systems. The exponential growth is consistent with the value of the real part of the eigenvalues calculated by the CEA with the linear model. The pattern of the velocity field shown in Fig. 9(b) is consistent with the deformed shape of the unstable mode 5 (cf. Fig. 4). At this stage all nodes are still in sliding and the contact nonlinearities due to sticking and detachment do not affect the system behavior.

The vibration amplitude increases progressively and when the tangential speed of the contact nodes reaches the peripheral speed of the rotating surface some of the nodes switch in sticking status. Afterwards (stage $C$ in Fig. 8), increasing the vibration amplitude the number of nodes in sticking and detachment condition increases and the system vibration reaches the limit cycle (stage D in Fig. 8). The nonlinearities due to the contact become relevant during this transition and produce a slight decrease of vibration frequency of the unstable mode, as shown by the spectrogram. In fact the appearance of zones of detachment with respect to the linear behavior introduces a less stiff constraint at the contact producing the decrease of the vibration frequency. The velocity field of the disc vibration during the limit cycle (cf. Fig. 9(c)) is not exactly the same of the modal shape of the mode 5, because the presence of sliding and detachment conditions is not accounted for in the linearized model used for the CEA. Super-harmonics appear at frequencies that are integer multiples of the original one, due to the nonlinear behavior of the system.

Fig. 10(a) shows the phase plot (displacement, velocity) of the node 1641 along the $x$ direction (cf. Fig. 1(b)). The stage B of the transient simulation is characterized by an increasing spiral, while the stage D is characterized by the stationary limit cycle. Fig. 10(b) presents the trace of the plane displacement of the contact node 1621 (cf. Fig. 1(b)), where the upper "vertical" line corresponds to the initial expansion of the inner rigid surface, the "horizontal" line corresponds to the stickslip phases and the arcs are due to the detachment of the node from the rotating surface. The amplitude increases until the system reaches the limit cycle.

\subsection{Transient analysis results for $\mu=0.3$ and $\omega=70 \mathrm{rpm}$}

This section presents the results of a transient analysis with the same boundary conditions, except for the rotational speed imposed to the inner rigid disc. In this case the rotational speed is $\omega=70 \mathrm{rpm}$.

Observing, as in the previous analysis, the acceleration response of a node of the disc (cf. Fig. 11), four stages can be highlighted, as identified with the letters from A to D.

The first part of transient response (stage A in Fig. 11) presents the same characteristics in terms of both modal shape and frequency $(5.8 \mathrm{kHz})$ of the simulation at $600 \mathrm{rpm}$. At time $t \simeq 0.013 \mathrm{~s}$ the spectrogram in Fig. 11 presents a frequency change. After this point the results of this simulation are quite different from the analysis presented above. As in the previous case, during stage B, the main harmonic content is at a frequency of about $9.9 \mathrm{kHz}$ that corresponds to the mode 5 of the CEA. The vibration amplitude grows until the tangential sliding speed of the contact nodes reaches the nil value. In this case, due to the reduced rotational speed of the rigid surface, only sticking nonlinearities (no detachments) appear at the contact during the steady state (stage D). In this case during the linear part of the transient response (stage B) there is a co-presence of two unstable modes, with evident beats in the response. On the contrary only one of the corresponding frequencies is present during the limit cycle when nonlinearities arise, in this case the main frequency recovered (10.5 kHz) corresponds to the 

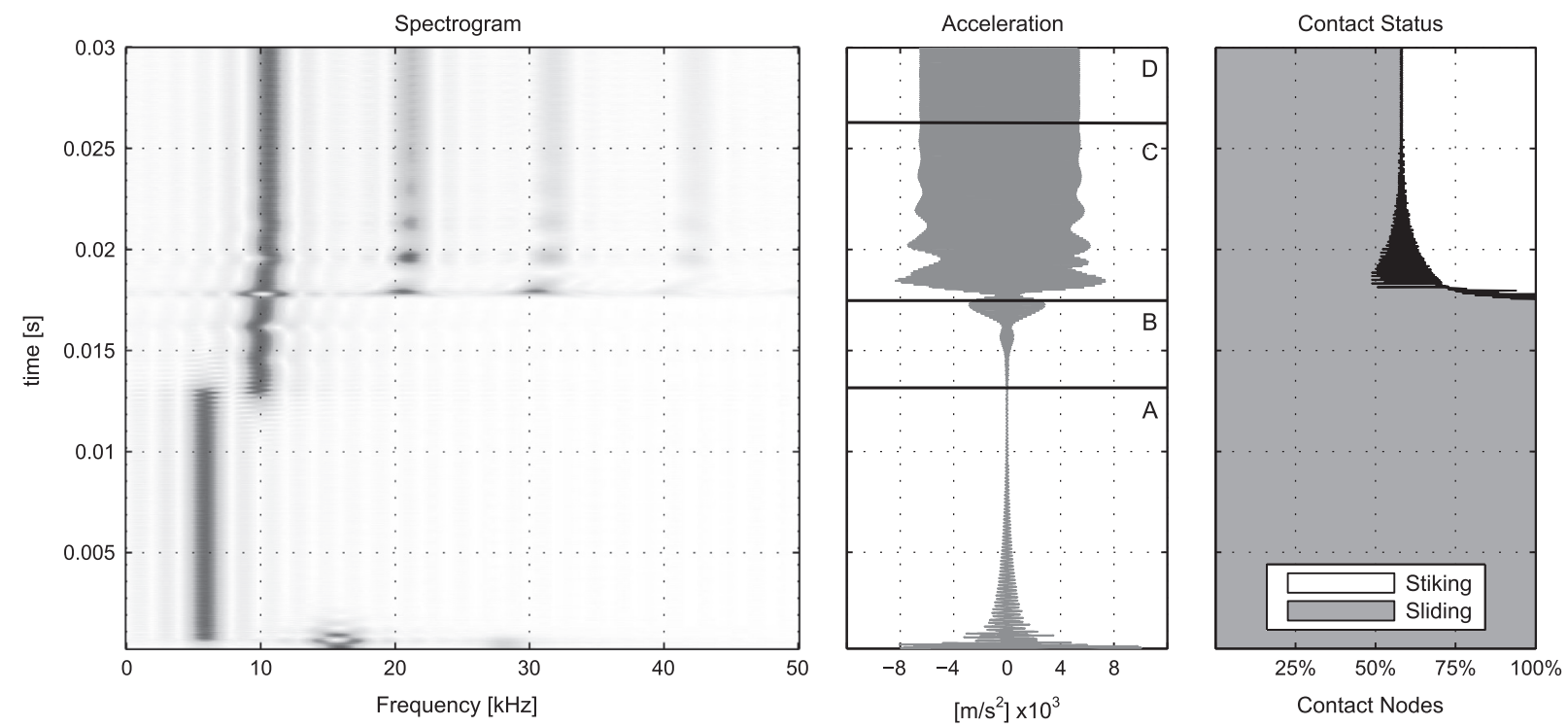

Fig. 11. Acceleration of an internal node with its spectrogram and status of the contact nodes over the time.

higher of the two unstable modes recovered in the linear part of the transient response and corresponds to another unstable mode (mode 7 calculated by the CEA).

The velocity distribution in this case is similar to modal shape of mode 7 confirming that at this rotational speed the system vibrates at another frequency due to the excitation of a different unstable system mode.

\subsection{Energy balance}

Energy analysis has been performed to observe the energy flows during the transient response of the system and to verify the energy balance. The total energy $\left(E_{T}\right)$ of the elastic media $\Phi$ is composed of the elastic potential energy $\left(E_{E}\right)$ and the kinetic energy $\left(E_{K}\right)$ :

$$
E_{T}=E_{E}+E_{K}=\frac{1}{2}\{x\}^{T}[K]\{x\}+\frac{1}{2}\{\dot{x}\}^{T}[M]\{\dot{x}\} .
$$

The inner rigid surface rotates with a constant rotational speed $\Omega$, and the external power can be expressed as the product of the torque $Q$ and the rotational speed $\Omega$ and it is equivalent to the integral over the whole contact interface $\partial \Phi$ of tangential stresses $\tau$ by the peripheral speed of the rigid surface $\Omega r$ :

$$
P_{E x}(t)=Q(t) \cdot \Omega=\int_{0}^{2 \pi} \tau(\phi, t) \cdot \Omega r \cdot d \phi
$$

The external power flow has three paths: (i) the power exchanged between the rigid surface and the elastic media by sticking $P_{S t}$; (ii) the power exchanged by sliding $P_{S l}$; (iii) the power dissipated at the contact $P_{D c}$ due to the relative velocity between the rigid and elastic contact surfaces when sliding occurs. Considering the tangential forces $T$ and the tangential velocity $v_{T}$ at the contact nodes of the FEM model, the three terms can be expressed as follows:

$$
P_{E x}=P_{S t}+P_{S l}+P_{D c}=\sum_{S t} T_{S t} \cdot v_{T, S t}+\sum_{S l} T_{S l} \cdot v_{T, S l}+\sum_{S l} T_{S l} \cdot\left(\Omega r-v_{T, S l}\right)
$$

where the first sum is extended to all the nodes in sticking $(S t)$ and $v_{T, S t}=\Omega r$, while the other two sums are extended to all nodes in sliding $(S l)$.

The internal forces due to the material damping $\left\{F_{D}\right\}=[C]\{\dot{x}\}$ produce an energy dissipation during a time step $(\delta t)$ that can be expressed as

$$
P_{D m}=\{\dot{x}\}^{T}[C]\{\dot{x}\} .
$$

The energy flows accounted for the energy balance extended to the elastic media $\Phi$ are the powers exchanged at the contact interface $\partial \Phi$ by sticking or sliding and the power dissipated in the elastic media by the material damping. The difference between the energy exchanged at the contact interface $(\partial \Phi)$ and the energy dissipated inside the body by material damping produces a variation of the total energy of the system. Furthermore, the variation of the total energy of the elastic body $\Phi$ is equal to the difference between the external power and the powers dissipated at the contact and inside the 

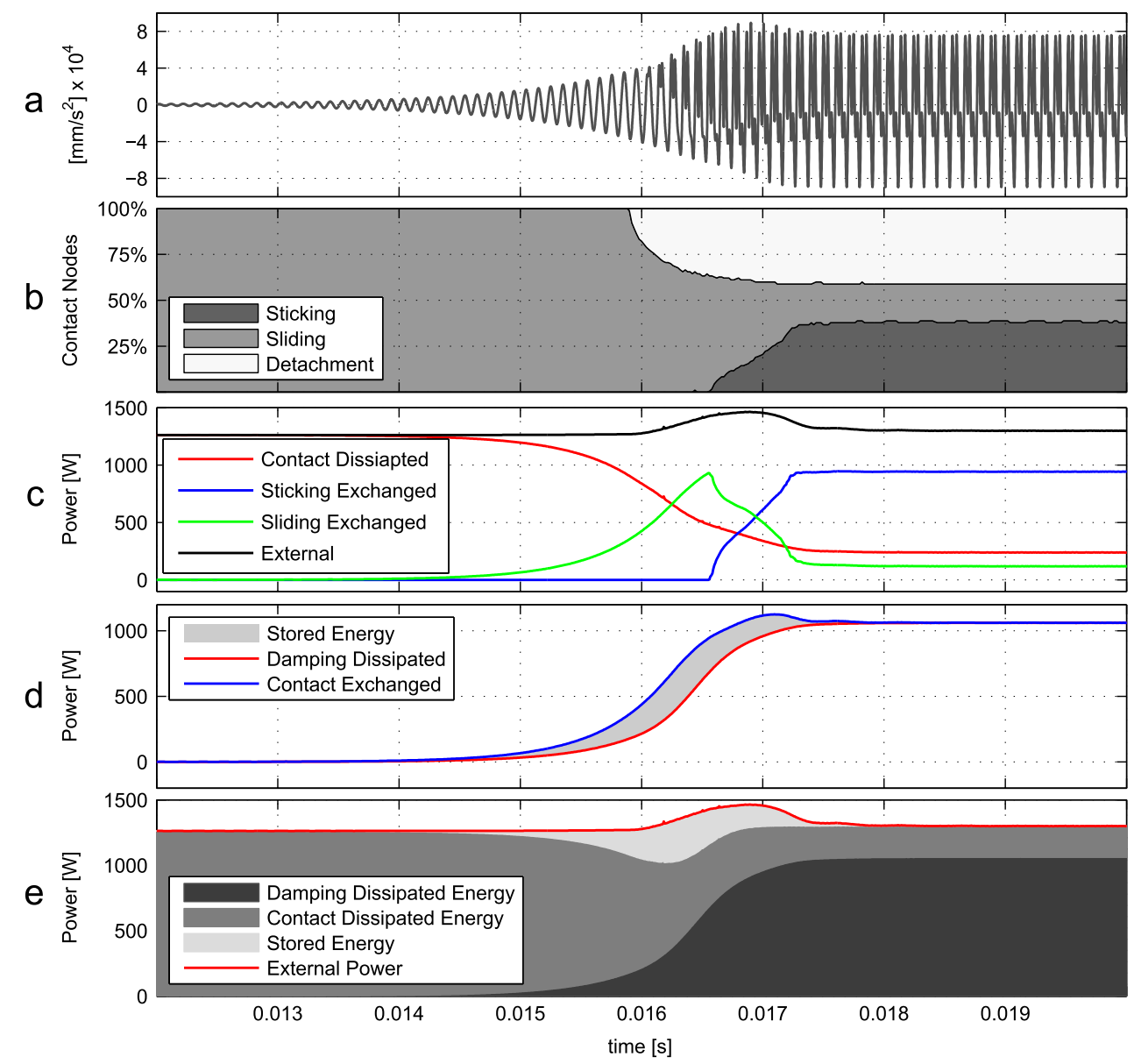

Fig. 12. Energy balance. (a) Acceleration response; (b) status of the contact nodes; (c) external power and decomposition into three terms: contact dissipated power, sliding power and sticking power; (d) power balance of the elastic body; (e) power balance among external power, damping dissipated power (by induced vibrations) and contact dissipated power, shaded areas represent the energies. (For interpretation of the references to color in this figure caption, the reader is referred to the web version of this paper.)

body by material damping:

$$
\frac{d E_{T}}{d t}=P_{S t}+P_{S I}-P_{D m}=P_{E x}-P_{D c}-P_{D m}
$$

When the system dynamics is unstable, the phase combination between the tangential force and tangential speed produces a flow of energy at the contact toward the elastic body, increasing its total energy content (elastic and kinetic energy). The higher the energy content is, the higher the vibration amplitude will be, which in fact increases when instabilities occur.

During the limit cycle the system reaches a dynamic equilibrium condition and the energy dissipated by material damping and contact balances the energy introduced at the contact.

Fig. 12 shows the quantities presented above during the simulation time. The different energy flows that constitute the external power are shown (cf. Fig. 12(c)) referred to the acceleration response (cf. Fig. 12(a)) and the status of contact nodes (cf. Fig. 12(b)).

The external power starts from a value that corresponds to the product of the peripheral speed of the rigid surface by the tangential contact stress due to the radial expansion in stable conditions. At the beginning of the simulation, when the system is near to its static equilibrium position, in overall sliding, the external power is entirely dissipated at the contact (black and red curves in Fig. 12(c)). When the unstable behavior appears (exponential growth of vibrations) there is a progressive increase of the energy that is introduced into the system by friction-induced-vibration (green curve) and a progressive reduction of the energy dissipated at the contact (red curve). The sum of these terms (black curve) is constant until the first detachments appear (cf. Fig. 12(b)). While at the beginning of the unstable vibrations the energy is introduced completely by sliding (green curve), when the sticking appears a drop of the sliding power curve can be noticed with an increase of the sticking term (blue curve). 


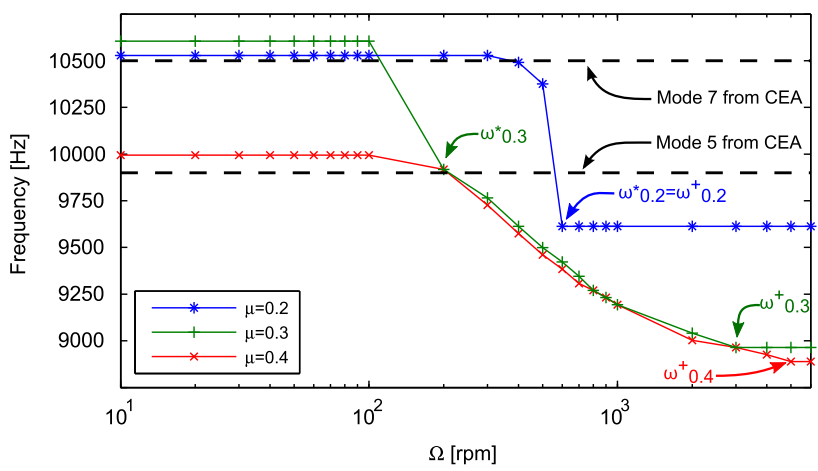

Fig. 13. Main frequencies recovered during the limit cycle for different values of the rotational speed $\Omega$ and friction coefficient $\mu$.

It can be noticed that the energy dissipated by the friction forces during the unstable behavior is less than the energy dissipated during the stable behavior (red curve in Fig. 12(c)). Fig. 12(c) shows the portion of the energy dissipated by friction at the contact and the energy dissipated by material damping in the bulk. At the beginning of the simulation the polycarbonate disc is sliding on the rigid surface without significant vibrations and almost all the external energy is dissipated at the contact; then, once the unstable vibrations occur, the external energy is dissipated by both material damping and frictional forces and the amount of energy dissipated at the contact decreases (cf. Fig. 12). This aspect is confirmed by experimental observations (see Section 6), that do not show overheating of the contacting interface during the unstable behavior.

During the limit cycle, the power dissipated at the contact (by frictional forces) is 18\% of the external power. The remaining part is divided into the Sticking power (72\%) and Sliding power (10\%), which are powers re-introduced by the contact into the polycarbonate disc as friction-induced vibrations and successively dissipated by material damping (82\% of the total external power).

Fig. 12(d) shows the energies introduced and dissipated by the elastic media (polycarbonate disc). The energy introduced (blue curve) is composed of the sum of sliding and sticking terms in Fig. 12(c), while the energy is dissipated by the material damping (red curve in Fig. 12(d)). The difference between the introduced and dissipated power (highlighted area in Fig. 12 (d)) represents the variation of the energy stored into the system by elastic and kinetic energy, due to the increase of the system vibrations. Once the limit cycle is reached, all the energy introduced into the polycarbonate disc is dissipated by material damping ( $82 \%$ of the total external power) and there is no more variation of the mechanical energy of the elastic media that vibrates with constant amplitude (limit cycle).

Furthermore, an increase of external energy needed to maintain a constant rotational velocity of the rigid surface can be noticed and the external power calculated during the unstable phase is higher than the external power calculated during the stable phase (black curve in Fig. 12(c)).

\subsection{Effect of rotational speed and local friction coefficient on transient solution}

When nonlinearities arise in a mechanical system, its behavior is not easily predictable. Observing the behavior of this system during the transient analysis, both rotational speed and friction coefficient are found to have relevant effects on the vibrational status reached during the limit cycle.

A parametric analysis has been performed to highlight the effect of both the rotational speed of the inner cylinder and the friction coefficient on the transient vibrational behavior of the polycarbonate disc.

In this study, 24 values of the rotational speed in the range 10-6000 rpm have been considered while the friction coefficient assumes the values $0.2,0.3$ and 0.4 . The main results in terms of the vibration frequency and status at the contact during the limit cycle are reported in Figs. 13 and 14.

Observing the trend of the vibration frequency (cf. Fig. 13) it can be noticed that by increasing the rotational speed, the vibration frequency generally decreases. For small values of the rotational speed ( $\Omega$ in the range $10-20 \mathrm{rpm}$ ) the main vibration frequency is comparable with the frequency of the unstable mode 7 (black dashed line in Fig. 13 at $10.5 \mathrm{kHz}$ ) obtained by CEA.

When the same unstable mode is excited (the unstable mode 7 in CEA, for $\Omega \leq 100 \mathrm{rpm}$ and friction coefficient equal to 0.2 and 0.3 ) the frequency increases by increasing the friction coefficient according to results of the parametric CEA in Fig. 6. At high rotational speed, when the nodes detachment status appears and the unstable vibration corresponds to the mode 5 in CEA, the frequency decreases with the increase of the rotational speed for friction coefficient equal to 0.3 and 0.4 , while it stays constant for friction coefficient equal to 0.2 . This is consistent with the increase of the percentage of nodes in detachment status showed in Fig. 14. The higher the friction coefficient is, the higher the percentage of nodes in detachment status will be, that produces a softening of the contact boundary condition. 

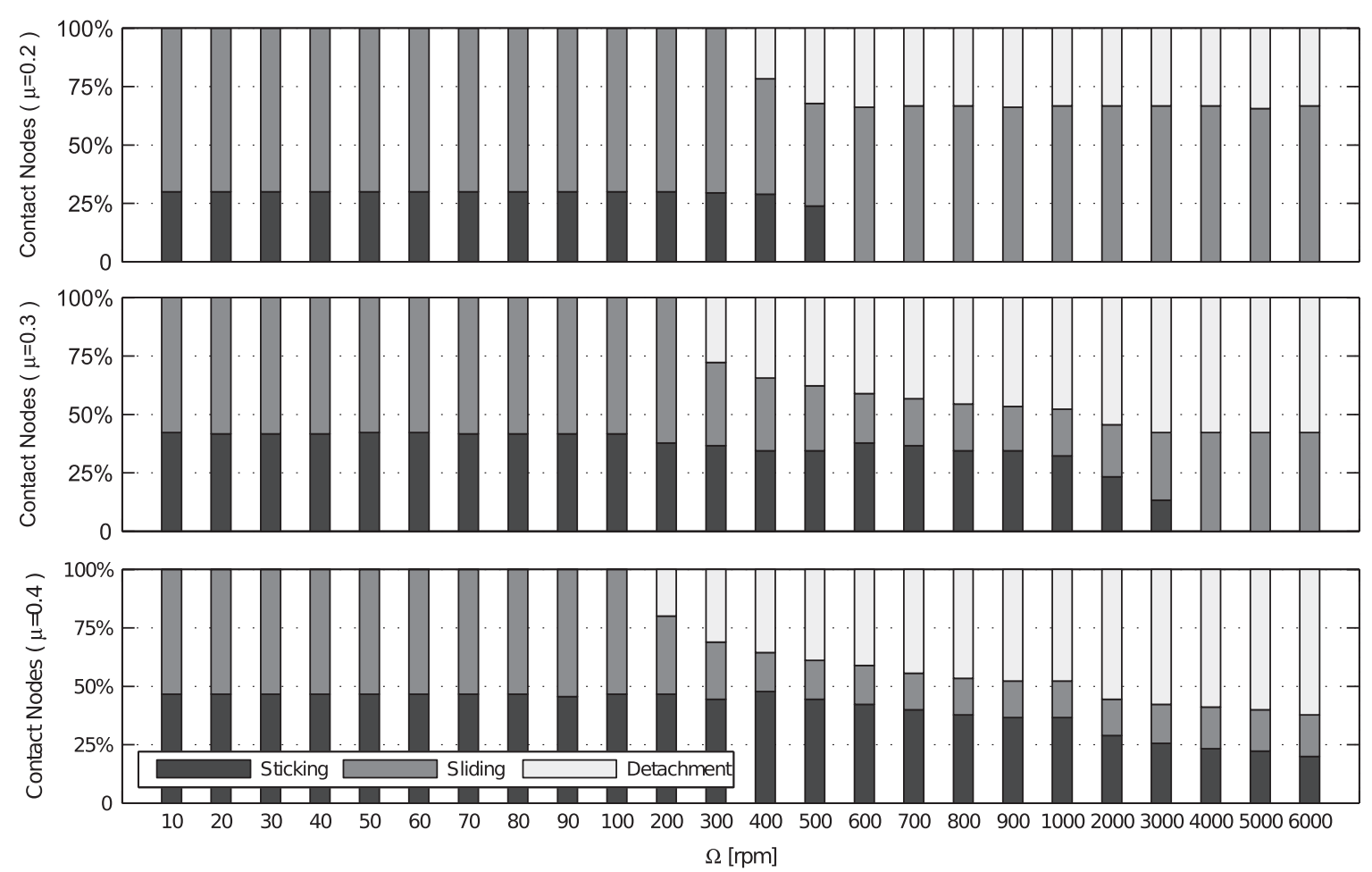

Fig. 14. Percentage of nodes in each status at the limit cycle for different values of friction coefficient $\mu$ and rotational speed $\Omega$.

In this case the frequency is lower than the one calculated by CEA with the linear system (black dashed line in Fig. 13 at $9.9 \mathrm{kHz}$ ), because at high rotational speed the system presents relevant portion of contact interface in detachment status, which causes a softening of the boundary conditions.

Fig. 14 shows the status of the contact interface during the limit cycle expressed as percentage of nodes in different statuses. For low values of rotational speed the contact status is initially divided between sliding and sticking. By increasing the rotational speed also detachment arises while the sticking tends to extinguish. These transitions move toward lower rotational speed for increasing friction coefficient due to the higher value of friction forces. Comparison between the results shown in Figs. 14 and 13 confirms that the reduction of the main frequency recovered during the limit cycle can be directly related to the increasing of the detachment zones on the contact interface.

Two characteristic values of rotational speed can be identified for each friction coefficient. The first one $\omega^{\star}$ is the value that produces a definitive selection of the unstable mode 5: it moves toward lower speed increasing the friction coefficient (cf. Fig. 13). The other characteristic value $\omega^{+}$indicates the minimum value of rotational speed that produces no further variation of the transient response. This is due to the stabilization (with the rotational speed) of the percentage of sticking, sliding and detachment zones. This occurs when no sticking zones are recovered at the contact interfaces, for the higher values of rotational speed $\Omega$ (cf. Fig. 14), and the sliding contact interaction does not take into account the value of relative speed but only its sign. Increasing the friction coefficient these characteristic values move toward higher values of rotational speed.

\section{Preliminary experimental validation of the numerical model}

The simplified geometry of the system allowed for developing an experimental set-up (cf. Fig. 1(a)) that is able to maintain contact and dynamic boundary conditions as constant as possible to reproduce dynamic instabilities [26].

In order to give a preliminary validation of the developed system, results of experimental tests are presented in Fig. 15 for a rotational speed of $45.58 \mathrm{rpm}$. The dynamic response of the system measured by an accelerometer along the radial and the tangential direction of an inner point of the polycarbonate disc is measured and analyzed.

The leftmost plot in Fig. 15 shows the acceleration measured during the test. The response is characterized by a large amplitude of harmonic vibration and sound emissions, with exponential increase up to the limit cycle. This confirms the onset of modal coupling instabilities in the experimental test.

The rightmost graph in Fig. 15 shows the spectrogram of the experimental response. Four main vibration frequencies are recovered at $6 \mathrm{kHz}, 7.5 \mathrm{kHz}, 9 \mathrm{kHz}$ and $16 \mathrm{kHz}$. In the authors' opinion these frequencies can be associated with the first four unstable modes found in CEA (cf. Table 1). Also superharmonics of the main frequencies can be detected due to the nonlinearity of the vibration status. 

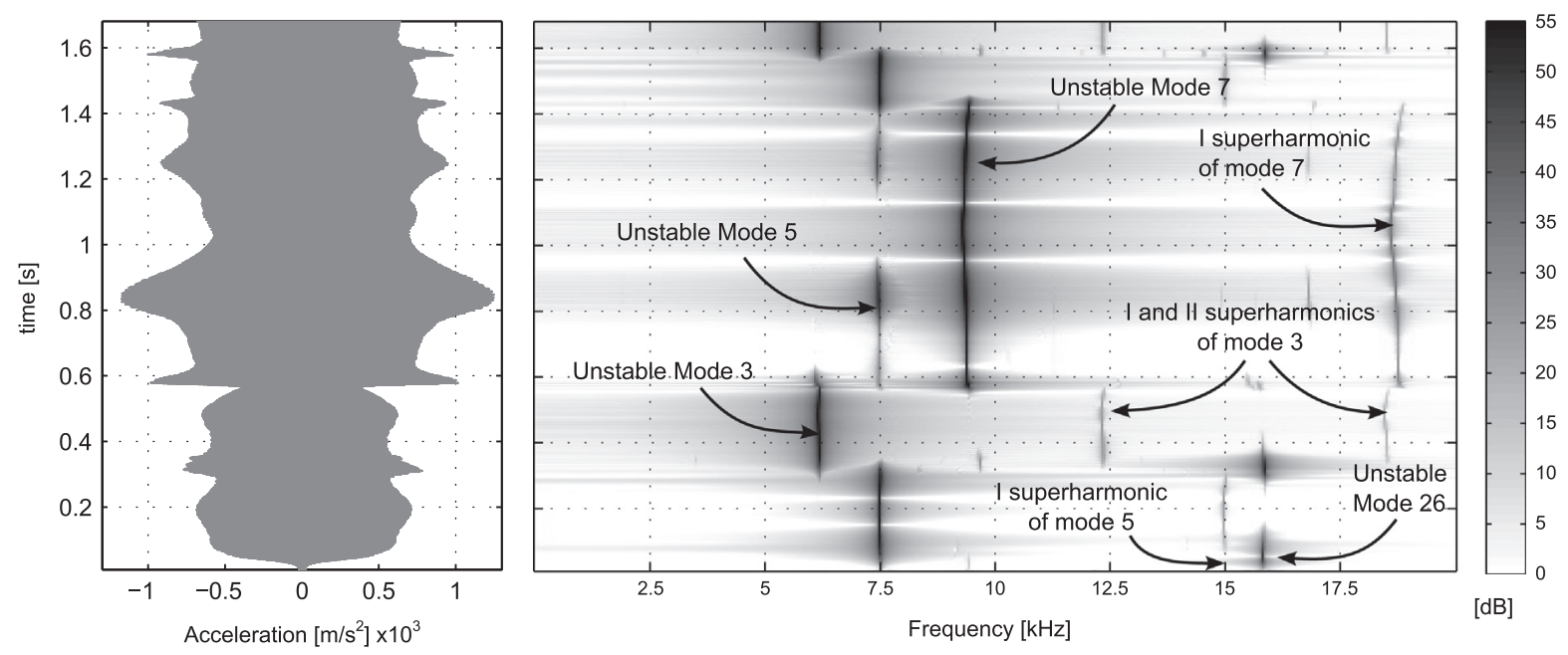

Fig. 15. Experimental in plane acceleration on the polycarbonate disc and signal spectrogram for $\Omega=45.58 \mathrm{rpm}$.

The difference between frequencies recovered experimentally and numerically is ascribed by the authors to the lower stiffness of the boundary conditions applied experimentally. While in the numerical model the constraint applied on the external edge of the polycarbonate disc is rigid, in the experiments the disc is clamped to the frame by three screws and a steel annular ring. This difference in frequency is confirmed by a numerical analysis on a 3D model that takes into account the real geometry and stiffness of the whole setup [27].

The occurrence of the dynamic instability is really sensitive to both the system dynamics and the contact conditions $[18,19,12]$ : due to the impossibility of obtaining exact symmetry in the experimental tests, the unstable vibrations do not reach a steady state condition as in the numerical analysis and a switching between the different unstable modes of the system is recovered experimentally (cf. Fig. 15).

Finally, thermal measurements on different points of the experimental setup did not indicate overheating of the contact interface during the occurrence of unstable vibrations. This is in agreement with the numerical results which show that during dynamic instabilities the energy is dissipated mainly in the bulk by material damping rather than locally at the contact by relative motion. These preliminary results suggest the need to investigate experimentally the temperature distribution in the bulk and next to the contact, in order to validate the numerical results and verify the possibility to use overheating as an indicator of contact and material damping.

The presented case is a preliminary test showing the capability of the designed system to provide squeal instabilities that can be used to validate the numerical simulations. Parametric analyses are needed to validate the numerical findings, and will be object of future works.

\section{Conclusions}

In this paper the behavior of a simple model, reproduced experimentally, has been numerically analyzed to investigate the contact dynamic instabilities due to mode coupling. The main advantage of the model is the accounting for a single deformable body in frictional contact with a rigid surface. The modes that are coupled by the frictional forces belong to the same component, namely the disc.

The numerical analyses showed good agreement between results from the linear CEA and the transient nonlinear analysis, allowing for a generalization of the mode-lock-in theory, which is usually investigated in the literature for brake squeal. The effects of the nonlinearities on the limit cycle are presented in detail.

Convergence analysis and comparison between the numerical results and experiments highlighted the main role played by material damping in selecting the dynamic response of the system. To obtain reliable results an experimental estimation of the material damping is necessary.

The simplicity of the model allows as well to develop a detailed energy analysis to distinguish between the energy introduced by the contact into the system and the energy dissipated by friction. The energy analysis allowed to quantify that part of the energy directly dissipated by the frictional forces at the contact and the energy that is first introduced into the system as friction-induced vibrations and subsequently dissipated by the material damping into the bulk.

The energy balance of the system showed that, during the limit cycle of the unstable vibrations the main part of the energy is dissipated by material damping. The energy dissipation at the contact during the contact dynamic instability is significantly lower than the dissipation during a stable sliding behavior. 
Experimental tests on the designed set-up showed the capability of the system to reproduce the in-plane modal instabilities. The introduction of correct values of the material damping allowed for predicting the amplitude of the friction induced vibrations, while the monitored temperature at the contact did not show detectable increase.

The performed parametric analysis highlights the main role of the energy flows on the selection of the unstable modes of vibration and it suggests a further energy investigation. The knowledge of the energy flows between contact interface and system can bring to a deeper understanding of friction-induced vibration phenomena to control unstable induced vibration or prevent surface degradation.

The power flow analysis, presented here for the specific issue of mode coupling instabilities, is a meaningful tool to distinguish between the energy dissipated at the contact (contact damping) and the energy that is introduced into the system as friction-induced vibrations and dissipated into the bulk by material damping.

\section{Acknowledgments}

Part of this work has been funded by the ANR (Agence National de la Recherche) program, in the framework of the ANRJCJC08-0020 DiNEET project (Dialogue Numérique Entre Echelles Tribologiques). The authors also wish to thank Prof. Laurent Baillet for his helpful suggestions and support.

\section{References}

[1] A. Akay, Acoustics of friction, J. Acoust. Soc. Am. 111 (4) (2002) 1525-1548, http://dx.doi.org/10.1121/1.1456514. URL: 〈http://link.aip.org/link/?JAS/111/ $1525 / 1\rangle$.

[2] M. Di Bartolomeo, A. Meziane, F. Massi, L. Baillet, A. Fregolent, Dynamic rupture at a frictional interface between dissimilar materials with asperities, Tribol. Int. 43 (9) (2010) 1620-1630. http://dx.doi.org/10.1016/j.triboint.2010.03.008 URL:〈http://www.sciencedirect.com/science/article/pii/ S0301679X10000721>.

[3] M. Di Bartolomeo, F. Massi, L. Baillet, A. Culla, A. Fregolent, Y. Berthier, Wave and rupture propagation at frictional bimaterial sliding interfaces: from local to global dynamics, from stick-slip to continuous sliding, Tribol. Int. 52 (2012) 117-131. http://dx.doi.org/10.1016/j.triboint.2012.03.008. URL: 〈http://www.sciencedirect.com/science/article/pii/S0301679X12001053〉.

[4] D. Tonazzi, F. Massi, A. Culla, L. Baillet, A. Fregolent, Y. Berthier, Instability scenarios between elastic media under frictional contact, Mech. Syst. Signal Process. 40 (2) (2013) 754-766. http://dx.doi.org/10.1016/j.ymssp.2013.05.022 URL: 〈http://www.sciencedirect.com/science/article/pii/S0888327013002811〉.

[5] M. Renouf, F. Massi, A. Saulot, N. Fillot, Numerical tribology of dry contact, Tribol. Int. 44 (7-8) (2011) 834-844.

[6] G. Sheng, Friction-Induced Vibrations and Sound: Principles and Applications, CRC press, Boca Raton, FL, 2007, ISBN-13:978-1420051797.

[7] R. Allgaier, L. Gaul, W. Keiper, K. Willner, Mode lock-in and friction modelling, in: International Conference on Computational Methods in Contact Mechanics, 1999, pp. 35-47.

[8] G. Chen, Z. Zhou, A self-excited vibration model based on special elastic vibration modes of friction systems and time delays between the normal and friction forces: a new mechanism for squealing noise, Wear 262 (2007) 1123-1139. http://dx.doi.org/10.1016/j.wear.2006.11.014 URL: 〈http://www. sciencedirect.com/science/article/pii/S0043164806004467〉.

[9] G. Chen, Z. Zhou, Experimental observation of the initiation process of friction-induced vibration under reciprocating sliding conditions, Wear 259 (2005) 277-281. http://dx.doi.org/http://dx.doi.org/10.1016/j.wear.2005.02.023 URL: 〈http://www.sciencedirect.com/science/article/pii/S0043164805001389〉.

[10] N. Coudeyras, J.-J. Sinou, S. Nacivet, A new treatment for predicting the self-excited vibrations of nonlinear systems with frictional interfaces: the constrained harmonic balance method, with application to disc brake squeal, J. Sound Vib. 319 (3) (2009) 1175-1199. http://dx.doi.org/10.1016/j.jsv. 2008.06.050 URL: 〈http://www.sciencedirect.com/science/article/pii/S0022460X08006111〉.

[11] F. Cantone, F. Massi, A numerical investigation into the squeal instability: effect of damping, Mech. Syst. Signal Process. 25 (5) (2011) 1727-1737. http:// dx.doi.org/10.1016/j.ymssp.2010.12.005 URL: 〈http://www.sciencedirect.com/science/article/pii/S0888327010004292〉.

[12] F. Massi, L. Baillet, O. Giannini, A. Sestieri, Brake squeal: linear and nonlinear numerical approaches, Mech. Syst. Signal Process. 21 (6) (2007) 2374-2393. http://dx.doi.org/10.1016/j.ymssp.2006.12.008 URL: 〈http://www.sciencedirect.com/science/article/pii/S0888327007000039〉.

[13] F. Massi, O. Giannini, Effect of damping on the propensity of squeal instability: an experimental investigation, J. Acoust. Soc. Am. 123 (4) (2008) 2017-2023. 10.1121/1.2875628 URL: 〈http://link.aip.org/link/?JAS/123/2017/1〉.

[14] F. Massi, O. Giannini, L. Baillet, Brake squeal as dynamic instability: an experimental investigation, J. Acoust. Soc. Am. 120 (3) (2006) 1388-1398. 10. 1121/1.2228745http://dx.doi.org/10.1121/1.222874510.1121/1.2228745 URL: 〈http://link.aip.org/link/?JAS/120/1388/1〉.

[15] A. Akay, O. Giannini, F. Massi, A. Sestieri, Disc brake squeal characterization through simplified test rigs, Mech. Syst. Signal Process. 23 (8) (2009) 2590-2607. http://dx.doi.org/10.1016/j.ymssp.2009.03.017 URL: 〈http://www.sciencedirect.com/science/article/pii/S088832700900082X).

[16] G. Chen, P. Wu, H. Dai, Z. Zhou, Comparative study on the complex eigenvalue prediction of brake squeal by two infinite element modeling approaches, Chin. J. Mech. Eng. 23 (2010) 8.

[17] F. Chevillot, J.-J. Sinou, N. Hardouin, Nonlinear transient vibrations and coexistences of multi-instabilities induced by friction in an aircraft braking system, J. Sound Vib. 328 (2009) 555-574. http://dx.doi.org/10.1016/j.jsv.2009.08.028 URL:〈http://www.sciencedirect.com/science/article/pii/ S0022460X09006865>.

[18] N. Kinkaid, O. O'Reilly, P. Papadopoulos, Automotive disc brake squeal, J. Sound Vib. 267 (1) (2003) 105-166. http://dx.doi.org/10.1016/S0022-460X(02) 01573-0 URL: 〈http://www.sciencedirect.com/science/article/pii/S0022460X02015730〉.

[19] H. Ouyang, W. Nack, Y. Yuan, F. Chen, Numerical analysis of automotive disc brake squeal: a review, Int. J. Veh. Noise Vib. 1 (3-4) (2005) 207-231, http: //dx.doi.org/10.1504/IJVNV.2005.007524. URL: 〈http://www.ingentaconnect.com/content/ind/ijvnv/2005/00000001/F0020003/art00003).

[20] H. Kasem, J.-F. Witz, P. Dufrénoy, Y. Desplanques, Monitoring of transient phenomena in sliding contact application to friction brakes, Tribol. Lett. 51 (2) (2013) 235-242, http://dx.doi.org/10.1007/s11249-013-0147-5. URL: 〈http://dx.doi.org/10.1007/s11249-013-0147-5〉.

[21] N. Hoffmann, M. Fischer, R. Allgaier, L. Gaul, A minimal model for studying properties of the mode-coupling type instability in friction induced oscillations, Mech. Res. Commun. 29 (4) (2002) 197-205. 〈http://dx.doi.org/10.1016/S0093-6413(02)00254-9〉 URL: 〈http://www.sciencedirect.com/ science/article/pii/S0093641302002549).

[22] J. Flint, J. Hultén, Lining-deformation-induced modal coupling as squeal generator in a distributed parameter disc brake model, J. Sound Vib. 254 (1) (2002) 1-21. http://dx.doi.org/10.1006/jsvi.2001.4052 URL: 〈http://www.sciencedirect.com/science/article/pii/S0022460X01940520〉.

[23] J.-J. Sinou, L. Jézéquel, Mode coupling instability in friction-induced vibrations and its dependency on system parameters including damping, Eur. J. Mech. A/Solids 26 (1) (2007) 106-122. http://dx.doi.org/10.1016/j.euromechsol.2006.03.002 URL: 〈http://www.sciencedirect.com/science/article/pii/ S099775380600026X>.

[24] L. Baillet, T. Sassi, Simulations numériques de différentes méthodes d'éléments finis pour les problèmes de contact avec frottement, C. R. Méc. 331 (11) (2003) 789-796. http://dx.doi.org/10.1016/j.crme.2003.08.005 URL: 〈http://www.sciencedirect.com/science/article/pii/S163107210300175X〉. 
[25] V. Linck, Modélisation numérique temporelle d'un contact frottant. mise en évidence d'instabiliés locales de contact-conséquences tribologiques (Ph. D. thesis), INSA de Lyon (2005).

[26] A. Saulot, F. Massi, M. Renouf, Y. Berthier, Competition between 3rd body flows and local contact dynamics, in: Proceedings of the Fifth World Tribology Congress (WTC-2013), 2013.

[27] M. Renouf, F. Massi, A. Saulot, N. Fillot, Dialogues numériques entre echelles tribologiques, Technical Report, INSA Lyon, 2011, 106 pp. http://dx.doi.org/ ANR-08-JCJC-0020.

[28] M. Godet, The third-body approach: a mechanical view of wear, Wear 100 (1-3) (1984) 437-452. http://dx.doi.org/10.1016/0043-1648(84)90025-5 URL: 〈http://www.sciencedirect.com/science/article/pii/0043164884900255〉.

[29] M. Godet, Third-bodies in tribology, Wear 136 (1) (1990) 29-45. doi:http://dx.doi.org/10.1016/0043-1648(90)90070-Q URL: 〈http://www.Sciencedir ect.com/science/article/pii/004316489090070Q).

[30] Y. Berthier, Maurice godet's third body, in: D. Dowson, C.M. Taylor, T.H.C. Childs, G. Dalmaz, Y. Berthier, L. Flamand, J.M. Georges, A.A. Lubrecht (Eds.), The Third Body Concept Interpretation of Tribological Phenomena, Tribology Series, vol. 31, Elsevier, Amsterdam, 1996, pp. 21-30. http://dx.doi.org/10.1016/ S0167-8922(08)70766-1 URL: 〈http://www.sciencedirect.com/science/article/pii/S0167892208707661〉.

[31] V.-H. Nhu, Dialogues numériques entre échelles tribologiques, These, INSA de Lyon (June 2013). URL: 〈http://tel.archives-ouvertes.fr/tel-00876855〉.

[32] M. Renouf, V.-H. Nhu, A. Saulot, F. Massi, First-body versus third-body: dialogue between an experiment and a combined discrete and finite element approach, J. Tribol. 136 (2) (2014) 021104-1/9. URL: 〈http://dx.doi.org/10.1115/1.4026062〉.

[33] L. Baillet, T. Sassi, Méthode d'éléments finis avec hybridisation frontière pour les problèmes de contact avec frottement, C. R. Math. 334 (10) (2002) 917-922. http://dx.doi.org/10.1016/S1631-073X(02)02356-7 URL: 〈http://www.sciencedirect.com/science/article/pii/S1631073X02023567〉.

[34] L. Baillet, T. Sassi, Mixed finite element formulation in large deformation frictional contact problem, Rev. Eur. Élém. 14 (2-3) (2005) 287-304, http: //dx.doi.org/10.3166/reef.14.287-304. arXiv:http://www.tandfonline.com/doi/pdf/10.3166/reef.14.287-304 URL: 〈http://www.tandfonline.com/doi/abs/ 10.3166/reef.14.287-304).

[35] N. Hoffmann, L. Gaul, Effects of damping on mode-coupling instability in friction induced oscillations, J. Appl. Math. Mech. 83 (8) (2003) 524-534, http://dx.doi.org/10.1002/zamm.200310022. URL: 〈http://dx.doi.org/10.1002/zamm.200310022〉.

[36] N.J. Carpenter, R.L. Taylor, M.G. Katona, Lagrange constraints for transient finite element surface contact, Int. J. Numer. Methods Eng. 32 (1) (1991) 103-128, http://dx.doi.org/10.1002/nme.1620320107. 